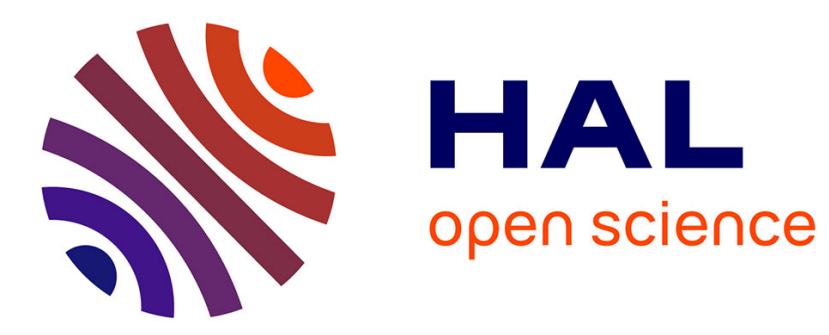

\title{
Toward fully conservative hybrid lattice Boltzmann methods for compressible flows
}

\author{
S. Zhao, G. Farag, Pierre Boivin, P. Sagaut
}

\section{To cite this version:}

S. Zhao, G. Farag, Pierre Boivin, P. Sagaut. Toward fully conservative hybrid lattice Boltzmann methods for compressible flows. Physics of Fluids, 2020, 32 (12), pp.126118. 10.1063/5.0033245. hal-03087980

\section{HAL Id: hal-03087980 \\ https://hal.science/hal-03087980}

Submitted on 24 Dec 2020

HAL is a multi-disciplinary open access archive for the deposit and dissemination of scientific research documents, whether they are published or not. The documents may come from teaching and research institutions in France or abroad, or from public or private research centers.
L'archive ouverte pluridisciplinaire HAL, est destinée au dépôt et à la diffusion de documents scientifiques de niveau recherche, publiés ou non, émanant des établissements d'enseignement et de recherche français ou étrangers, des laboratoires publics ou privés. 


\section{Toward fully conservative hybrid Lattice Boltzmann Methods for compressible flows}

S. Zhao (赵崧), ${ }^{1,2}$ G. Farag, ${ }^{1}$ P. Boivin, ${ }^{1,}$ (a) and P. Sagaut ${ }^{1}$

1) Aix Marseille Univ, CNRS, Centrale Marseille, M2P2, 13451 Marseille, France

${ }^{2)}$ CNES launchers directorate, Paris, France

(Dated: December 15, 2020)

This article presents a new numerical scheme designed to solve for any scalar equation coupled with a Lattice-Boltzmann solver (in so-called hybrid methods). Its most direct application is to solve an energy equation, in parallel with a Lattice-Boltzmann solver dealing with mass and momentum conservation.

The numerical scheme is specifically designed to compute the energy flux consistently with the mass and momentum flux (as is done, for instance, using Riemann solvers).

This scheme effectively lifts a major limitation of current compressible hybrid Lattice-Boltzmann, in which the energy conservation is tackled under nonconservative form, leading to discretization errors on jump conditions across shocks.

Combined with our recently presented pressure-based solver [G. Farag et al, Physics of Fluids, vol. 32, no. 6, p. 066106, (2020)], the resulting hybrid Lattice-Boltzmann scheme is, to the authors' knowledge, the first to numerically conserve simultaneously mass, momentum and total energy.

a) Electronic mail: pierre.boivin@univ-amu.fr 


\section{INTRODUCTION}

Lattice Boltzmann Methods (LBM) has received a fastly growing interest in the past two decades. They are now recognized as a powerful tool for Computational Fluid Dynamics, and as a potential candidate for a breakthrough toward revolutionary $C F D^{1}$. Most published works dealing with LBM are related to low Mach number continuum flows, covering a broad range of flow physics, among which multiphase flows, thermal flows, combustion and non-Newtonian flows ( $\sec ^{2 \sqrt{3}}$ for examples). Therefore, designing an efficient Lattice Boltzmann Method well suited for viscous high speed subsonic and supersonic flows remains a challenging issue, since the Asymptotic Preservation (AP) as defined by Filbet and Jin $\frac{45}{45}$ for this case, i.e. the recovery of the compressible Navier-Stokes equations is tricky (see, e.g. ${ }^{6}[9]$ for examples dealing with AP kinetic schemes). It is reminded here that a Lattice Boltzmann Method has the AP property, if, at fixed mesh size and time step, a consistent discretization of the Navier-Stokes equations is recovered in the limit of vanishing Knudsen number.

Since LBM can be derived as a discretization of the Boltzmann equation 10 , it can inherit from the later the capability to handle compressible flow physics. Here, the problem lies in the efficiency of LBM rather than in an intrinsic inability to capture compressible flows (assuming that the conservativity of the method has been enforced), since to recover accurately compressible flow physics a direct and "rough" discretization of the Boltzmann equation in velocity, space and time is known to lead to the definition of numerical methods with non-standard lattices that may involve a large number of discrete velocities ${ }^{3[10}$.

A common way to decrease the computational cost and the memory storage is to use a segregated approach, in which the energy equation is treated separately from the mass and momentum conservation equations. Doing so, it is expected to that it is possible to drastically reduce the number of unknowns per cell (i.e. the number of discrete velocities) along with a decrease of the algorithmic complexity of the equation associated to each unknown (i.e. the computational complexity of the collision model). On the opposite, in monolithic approaches all equations are treated simultaneously in the same way, e.g.11 17.

Segregated Lattice Boltzmann methods can be grouped into two main classes. The first one corresponds to Double Distribution Function (DDF) approach in which a second set of distribution functions is defined to solve the energy equation via a kinetic advection- 
relaxation approach, e.g.18 21 . The second one is related to hybrid methods, in which the macroscopic energy equation is solved using a classical Finite Volume/Difference/Element method, e.g.22 24 . Both approaches have shown promising results in the recent past, and are being actively developed by various research groups.

Segregated methods, as all methods based on a discretization of the Boltzmann equation, should be Asymptotic Preserving (AP) methods and therefore should be conservative. The conservativity issue has been addressed by several authors for methods originating in Boltzmann kinetic equation (e.g., sec 25 ), and it appears that several features of usual LBM can lead to the loss of conservativity:

- Since LB methods are discrete ordinate/velocity methods with fixed discrete velocities, their collision models must be adapted in order to get an exact/conservative evaluation of the moments of interest and the preservation of the associated collision invariants, while still recovering the targeted macroscopic equations. Adaptation of the collision kernels can be done in several ways, among which supplementing a truncated polynomial expansion of the Maxwellian equilibrium function found in gas kinetic theory by forcing terms to balance truncation errors (e.g. $\frac{18|19| 21}{23}$ ), or building a synthetic collision model that explicitly enforces some constrains (usually solving a nonlinear problem at every grid point and time step), e.g!1112]14 16/20|29]30. It is worth noting that in the former case, many authors refer to conservativity error as Galilean invariance error or symmetry-breaking errors, since the error scales as a power of the velocity.

- Time integration, including the splitting between the streaming and the collision steps, must be performed in a carefully chosen way, e.g. ${ }^{25}$.

To the knowledge of the authors, a complete theoretical analysis of the AP and conservation properties of segregated LBM for compressible viscous flows is still missing. Segregated hybrid LBM for thermal compressible flows renders the problem theoretically more difficult, since it mixes a mesoscopic approach for mass and momentum conservation with a macroscopic approach for energy. Numerical experiments have shown that it is a powerful approach, but recent theoretical analyses and numerical experiments ${ }^{31}$ have shown that some conservativity issues may arise that can lead to limitations in terms of numerical stability and convergence toward the correct shock jump relation for strong shock waves. More pre- 
cisely, a lack of consistency in the mass flux $\rho \mathbf{u}$ evaluation between the mass and momentum equation on one hand, and the energy equation on the other hand has been identified.

This problem can be partially cured by using a non-conservative form of the energy equation and choosing a thermodynamic variable whose evolution equation does not explicitly exhibit a pressure work term $p \nabla \cdot \mathbf{u}$, e.g. the entropy as in 23 . While increasing the stability of the method, this choice doesn't lead to a fully conservative method, and Rankine-Hugoniot shock jump relations are not exactly recovered for strong shock waves. This can be understood considering the advection of a conserved scalar quantity $\phi$ in parallel of the mass conservation equation:

$$
\begin{gathered}
\left(\frac{\partial \rho}{\partial t}\right)_{I}+(\nabla \cdot(\rho \mathbf{u}))_{I}=0 \\
\left(\frac{\partial \rho \phi}{\partial t}\right)_{I I}+(\nabla \cdot(\rho \mathbf{u} \phi))_{I I}=0
\end{gathered}
$$

where subscripts I and II refer to LBM and macroscopic discretization, respectively. The last conservative equation can be rewritten as a combination of a non-conservative equation and the mass conservation equation

$$
\left(\rho \frac{\partial \phi}{\partial t}+\phi \frac{\partial \rho}{\partial t}\right)_{I I}+(\phi \nabla \cdot(\rho \mathbf{u})+\rho \mathbf{u} \cdot \nabla \phi)_{I I}=0
$$

from which one obtains

$$
\left[\rho\left(\frac{\partial \phi}{\partial t}+\mathbf{u} \cdot \nabla \phi\right)\right]_{I I}=-\left[\phi\left(\frac{\partial \rho}{\partial t}+\nabla \cdot(\rho \mathbf{u})\right)\right]_{I I}
$$

showing that the scalar is conserved and the conservative and non-conservative forms of the scalar equation are equivalent if the right hand side of Eq. (4) is null. Since the mass flux is estimated via Eq. (1), one can see that an error proportional to $\phi\left[(\nabla \cdot(\rho \mathbf{u}))_{I}-(\nabla \cdot(\rho \mathbf{u}))_{I I}\right]$ appears in hybrid methods based on a conservative form of the scalar equation.

The present paper aims at designing a fully conservative hybrid LBM approaches for thermal compressible flows that will satisfy the AP condition for the continuum compressible regime, including solutions with shock waves. To this end, a new discretization of the macroscopic thermodynamic variable equation is introduced, that will allow for the use of the conservative form of the total energy, resulting in a dramatic improvement of both the conservativity and stability of the method. It relies on the idea that the macroscopic and the 
kinetic equation must be coupled in a deeper way than in previous hybrid LBM approaches, in order to alleviate the inconsistency in the mass flux evaluation between the two parts of the global method. To this end, it is proposed to evaluate the fluxes in the macroscopic equation for the thermodynamic variable directly from the distribution functions of the kinetic part. The idea of using a kinetic approach to evaluate numerical fluxes in a macroscopic evolution equation is not new. As a matter of fact, it is the cornerstone of Gas Kinetic Schemes (GKS) proposed during the 1970's for Euler and Navier-Stokes equations (sec $32 \sqrt[37]{37}$ ) and

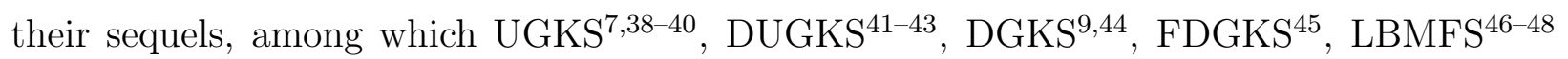
and SLBM 192 . All these approaches are monolithic ones, and therefore they cannot be used straightforwardly to improve segregated hybrid LBM.

In a separate way, some authors have proposed so-called Lattice Boltzmann schemes for scalar transport $\frac{53}{57}$, with application to the transport of stresses in non-Newtonian fluid models and passive scalar transport. In these schemes, the advection term for the scalar quantity is directly computed by combining the distribution functions and the scalar quantity, rather than computing the macroscopic velocity and then applying a usual Finite Difference/Finite volume Scheme to solve the macroscopic equation. An important point is that these schemes have all been derived to recover the non-conservative form of the scalar equation, assuming flow incompressibility.

In order to improve conservativity and recover AP property for the continuum compressible flow regime, it is proposed in the present paper to improve the previously proposed hybrid LBM methods by extending the so-called Lattice Boltzmann scalar schemes to energy equation. A main novelty of the present method is that mass and momentum equation are solved in a classical LBM way, while a macroscopic evolution equation is still used for energy, the fluxes of the later being evaluated from distribution functions. To the knowledge of the authors, such a method has not been proposed yet.

This paper is organized as follows: the scalar transport schemes with LBM populations will be presented in Section II. Conservation properties and stability are assessed using the classical Taylor-Green Vortex (TGV) test case in Section IV. Compressible test cases with shocks are then addressed in Section $\nabla$ to demonstrate the performance of the schemes when discontinuities are encountered. Conclusions are drawn in Section VI. 


\section{SCALAR TRANSPORT USING LBM DISTRIBUTION FUNCTIONS}

\section{A. Scalar flux schemes using collision populations}

The present idea of using LBM populations for scalar transport comes from the Taylor analysis of LBM schemes. The streaming step of almost any LBM scheme can be expressed as

$$
f_{i}(\boldsymbol{x}, t+\Delta t)=f_{i}^{\mathrm{col}}\left(\boldsymbol{x}-\boldsymbol{c}_{\boldsymbol{i}} \Delta t, t\right)
$$

where $f_{i}$ is the population and $f_{i}^{\text {col }}$ the collide population to be streamed ${ }^{3}$, and $\boldsymbol{c}_{\boldsymbol{i}}$ are the discrete velocities of the considered DnQm lattice. Their detailed expressions depend on the LBM scheme, but Eq. (5) always gives the transport equation for the moments of $f_{i}$

$$
\Pi_{\alpha_{1} \cdots \alpha_{n}}^{f} \equiv \sum_{i} c_{i \alpha_{1}} \cdots c_{i \alpha_{n}} f_{i}
$$

through Taylor expansion, where the vector $\boldsymbol{c}_{\boldsymbol{i}}$ denotes the lattice velocity of direction $i$. Multiplying Eq. (5) by the solution-independent term $c_{i \alpha_{1}} c_{i \alpha_{2}} \cdots c_{i \alpha_{n}}$ and then writting the Taylor series expansion of its R.H.S around $\boldsymbol{x}$, one obtains

$$
\begin{aligned}
\Pi_{\alpha_{1} \cdots \alpha_{n}}^{f}(\boldsymbol{x}, t+\Delta t) & =\sum_{i} c_{i \alpha_{1}} \cdots c_{i \alpha_{n}} f_{i}^{\mathrm{col}}\left(\boldsymbol{x}-\boldsymbol{c}_{\boldsymbol{i}} \Delta t, t\right) \\
= & \prod_{\alpha_{1} \cdots \alpha_{n}}^{f^{\mathrm{col}}}-\Delta t \frac{\partial}{\partial x_{\alpha_{n+1}}} \prod_{\alpha_{1} \cdots \alpha_{n+1}}^{f^{\mathrm{col}}} \\
& \quad+\frac{\Delta t^{2}}{2} \frac{\partial^{2}}{\partial x_{\alpha_{n+1}} \partial x_{\alpha_{n+2}}} \prod_{\alpha_{1} \cdots \alpha_{n+2}}^{f^{\mathrm{col}}}+\mathcal{O}\left(\Delta t^{3}\right),
\end{aligned}
$$

where the variables without any space or time indicators denote the corresponding values at $(\boldsymbol{x}, t)$ for the sake of simplicity. This equation indicates a numerical scheme for the divergence of moments

$$
\begin{aligned}
\nabla^{L} \cdot\left(\Pi_{\alpha_{1} \cdots \alpha_{n+1}}^{\mathrm{col}}\right) & \equiv \frac{1}{\Delta t}\left(\prod_{\alpha_{1} \cdots \alpha_{n}}^{f^{\mathrm{col}}}-\sum_{i} c_{i \alpha_{1}} \cdots c_{i \alpha_{n}} f_{i}^{\mathrm{col}-}\right) \\
& =\frac{\partial}{\partial x_{\alpha_{n+1}}} \prod_{\alpha_{1} \cdots \alpha_{n+1}}^{f^{\mathrm{col}}}-\frac{\Delta t}{2} \frac{\partial^{2}}{\partial x_{\alpha_{n+1}} \partial x_{\alpha_{n+2}}} \Pi_{\alpha_{1} \cdots \alpha_{n+2}}^{f^{\mathrm{col}}}+\mathcal{O}\left(\Delta t^{2}\right)
\end{aligned}
$$

where the upper script ${ }^{-}$denote the variables at $\left(\boldsymbol{x}-\boldsymbol{c}_{\boldsymbol{i}} \Delta t, t\right)$. For instance, the spatial derivative of the first order moments of the collide population, usually the momentum $\Pi_{\alpha} \equiv$ 
$\sum_{i} c_{i \alpha} f_{i}^{\mathrm{col}}=\rho u_{\alpha}$, can be evaluated as

$$
\nabla^{L} \cdot(\rho \boldsymbol{u}) \equiv \frac{1}{\Delta t} \sum_{i}\left(f_{i}^{\mathrm{col}}-f_{i}^{\mathrm{col}-}\right)=\frac{\partial}{\partial x_{\alpha}} \rho u_{\alpha}-\frac{\Delta t}{2} \frac{\partial^{2}}{\partial x_{\alpha} \partial x_{\beta}} \Pi_{\alpha \beta}^{f^{\mathrm{col}}}+\mathcal{O}\left(\Delta t^{2}\right),
$$

seemingly a first-order approximation. Yet, combining this equation with Eq. (7) for next order moment $\Pi_{\alpha \beta}$ yields

$$
\Pi_{\alpha_{1} \cdots \alpha_{n}}^{f}(t+\Delta t, \boldsymbol{x})=\Pi_{\alpha_{1} \cdots \alpha_{n}}^{f^{\mathrm{col}}}-\frac{\Delta t}{2} \frac{\partial}{\partial x_{\alpha_{n+1}}}\left[\Pi_{\alpha_{1} \cdots \alpha_{n+1}}^{f^{\mathrm{col}}}+\Pi_{\alpha_{1} \cdots \alpha_{n+1}}^{f}(\boldsymbol{x}, t+\Delta t)\right]+\mathcal{O}\left(\Delta t^{3}\right),
$$

showing that the numerical operator $\nabla^{L}$ is second-order accurate for the LBM mass flux evaluation, without any artificial viscous terms

$$
\nabla^{L} \cdot(\rho \boldsymbol{u})=\frac{1}{2}\left[\frac{\partial}{\partial x_{\alpha}} \rho u_{\alpha}+\frac{\partial}{\partial x_{\alpha}} \rho u_{\alpha}(t+\Delta t, \boldsymbol{x})\right]+\mathcal{O}\left(\Delta t^{2}\right) .
$$

It is worth noting that the above equation just serves as a demonstration of the order of accuracy in the evaluation of the mass flux in LBM. In practice, values at the next time step $t+\Delta t$ are never used, i.e. the LBMs considered in the current study are always explicit. More illustrative deductions of the above equations can be found in appendix A.

Let us now consider the multiplication of a scalar field $\phi$ and the collide population $f^{\text {col }}$, denoted by

$$
f_{i}^{\phi} \equiv \phi f_{i}^{\mathrm{col}}
$$

whose moments are

$$
\Pi_{\alpha_{1} \cdots \alpha_{n}}^{f^{\phi}} \equiv \sum_{i} c_{i \alpha_{1}} \cdots c_{i \alpha_{n}} \phi f_{i}^{\mathrm{col}}=\sum_{i} \phi \prod_{\alpha_{1} \cdots \alpha_{n}}^{f^{\mathrm{col}}}
$$

Inserting the first moments of $f_{i}^{\phi}$ into Eq. (8), one obtains the following numerical scheme for the scalar flux

$$
\begin{aligned}
\nabla^{U} \cdot(\rho \boldsymbol{u} \phi) & \equiv \frac{1}{\Delta t} \sum_{i}\left(f_{i}^{\phi}-f_{i}^{\phi^{-}}\right) \\
& =\frac{\partial}{\partial x_{\alpha}} \rho u_{\alpha} \phi-\frac{\Delta t}{2} \frac{\partial^{2}}{\partial x_{\alpha} \partial x_{\beta}} \Pi_{\alpha \beta}^{f^{\phi}}+\mathcal{O}\left(\Delta t^{2}\right)
\end{aligned}
$$

Unfortunately, this scheme is only first order accurate with a significant artificial viscous term proportional to the second moments of $f^{\phi}$. This dissipation term can not be canceled by time marching like the mass flux. Indeed, unlike the mass flux, the scalar flux $\rho u_{\alpha} \phi$ is not 
transported by the population $f^{\phi}$ anymore. So that Eq. 10 can not be applied to cancel the artificial viscous terms, as discussed in Appendix A.

However, Some modifications can be performed to make the flux more "central" in order to reduce the numerical dissipation. Instead of using fully upwind reconstruction of $\phi$, one can use a centered reconstruction the scalar, leading to the following numerical divergence operator

$$
\nabla^{C} \cdot(\rho \boldsymbol{u} \phi) \equiv \frac{1}{\Delta t} \sum_{i}\left[f_{i}^{\mathrm{col}} \frac{\phi^{+}+\phi}{2}-f_{i}^{\mathrm{col}^{-}} \frac{\phi+\phi^{-}}{2}\right]
$$

where the upper script ${ }^{+}$denotes the variables at $\left(\boldsymbol{x}+\boldsymbol{c}_{\boldsymbol{i}} \Delta t, t\right)$. The Taylor expansion of this equation given in Appendix B shows that this centered scheme does not bring any artificial viscous terms with respect to the scalar field.

It is important noting that the terms "upwind" or "centered" in this context are defined with respect to the lattice velocities. In practice, both $\nabla^{U}$ and $\nabla^{C}$ operators use the values of the scalar and collision populations from all neighbor nodes in the lattice, which is quite different from classical upwind schemes.

It is worth mentioning that in both numerical divergence operators $\nabla^{U}$ and $\nabla^{C}$, the mass flux part in the scalar flux is numerically identical to the mass flux resolved by the LBM. These numerical schemes are computationally affordable under the hybrid LBM-FD framework, because the collision population is already available. Indeed, these scalar flux operators are just special forms of finite difference method (FDM) except that, the coefficients are now accounting for the mass transport. In other words, the operators proposed here are just special forms of FD spatial derivation schemes which intend to inherit some numerical properties of double distribution approaches, with a reduced computational cost.

\section{B. Bridging with other LBM scalar transport schemes}

The idea of using LBM populations to compute the scalar transport can be found in

several papers, sec ${ }^{53} \sqrt[55]{5}$. Generally speaking, these works focus on the transport of a passive scalar in non-conservative form, by evaluating the $\boldsymbol{u} \cdot \nabla \phi$ term using the LBM populations from neighboring cells. For instance, Onishi et al. ${ }^{53}$ consider that the mass transport from cell $\boldsymbol{x}$ to $\boldsymbol{x}+\boldsymbol{c}_{\boldsymbol{i}} \Delta t$ by streaming is equal to $f_{i}^{\text {col }}(\boldsymbol{x})$; and that the one from cell $\boldsymbol{x}+\boldsymbol{c}_{\boldsymbol{i}} \Delta t$ to $\boldsymbol{x}$ is given by $f_{\bar{i}}^{\operatorname{col}}\left(\boldsymbol{x}+\boldsymbol{c}_{\boldsymbol{i}} \Delta t\right)$, where the index $\bar{i}$ corresponds to the lattice whose direction is 
opposite to lattice $i$. Thus, the scalar transport is modeled as

$$
(\boldsymbol{u} \cdot \nabla \phi)^{O} \equiv \frac{1}{\Delta t} \sum_{i}\left[\frac{\phi f_{i}^{\mathrm{col}}}{\rho}(\boldsymbol{x})-\frac{\phi f_{\bar{i}}^{\mathrm{col}}}{\rho}\left(\boldsymbol{x}+\boldsymbol{c}_{\boldsymbol{i}} \Delta t\right)\right],
$$

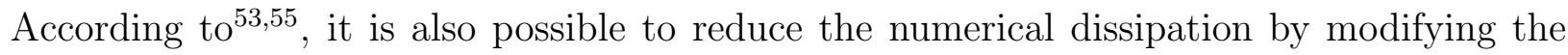
population used to transport the scalar :

$$
(\boldsymbol{u} \cdot \nabla \phi)^{O C} \equiv \frac{1}{\Delta t} \sum_{i}\left\{\left[\phi\left(\frac{f_{i}^{\text {col }}}{\rho}-\omega_{i}\right)\right](\boldsymbol{x})-\left[\phi\left(\frac{f_{\bar{i}}^{\text {col }}}{\rho}-\omega_{\bar{i}}\right)\right]\left(\boldsymbol{x}+\boldsymbol{c}_{\boldsymbol{i}} \Delta t\right)\right\},
$$

Other authors ${ }^{54}$ noticed that the net mass exchange through the interface $\boldsymbol{x}+\frac{1}{2} \boldsymbol{c}_{\boldsymbol{i}} \Delta t$ into cell $\boldsymbol{x}$ is equal to

$$
\Delta m_{i} \equiv f_{\bar{i}}^{\mathrm{col}}\left(\boldsymbol{x}+\boldsymbol{c}_{\boldsymbol{i}} \Delta t\right)-f_{i}^{\mathrm{col}}(\boldsymbol{x})
$$

so that the total scalar exchange can be expressed by only considering the "upstream" values, leading to

$$
(\boldsymbol{u} \cdot \nabla \phi)^{O S} \equiv \sum_{i} \frac{-\Delta m_{i}}{\rho(\boldsymbol{x}, t+\Delta t)} \begin{cases}\phi(\boldsymbol{x}, t) & \text { if } \Delta m_{i} \leq 0 \\ \phi\left(\boldsymbol{x}+\boldsymbol{c}_{\boldsymbol{i}} \Delta t, t\right) & \text { otherwise }\end{cases}
$$

It is worth noting that these numerical schemes for non-conservative scalar flux cannot be applied directly in compressible flows. As a matter of fact, one can demonstrate that these schemes rely on the incompressibility assumption, i.e. $\nabla \cdot \boldsymbol{u} \approx 0$, to work properly. In order to compare these schemes with the $\nabla^{U}$ and $\nabla^{C}$ operators in compressible configurations, their conservative extensions given in Appendix Clare used as references in the present work.

\section{Applications in the energy transport for HRR-p LBM}

As mentioned in the introduction, the scalar transport methods designed in the current work aim at curing the incompatibility between mass flux evaluations in the energy and mass conservation equations in hybrid LBM-FD approaches. For instance, in HRR- $p$ LBM proposed in $\sqrt{58}$, the governing equations for the flow fields are

$$
\begin{aligned}
& \frac{\partial \rho}{\partial t}+\frac{\partial \rho u_{\alpha}}{\partial x_{\alpha}}=0 \\
& \frac{\partial \rho u_{\alpha}}{\partial t}+\frac{\partial\left(\rho u_{\alpha} u_{\beta}+p \delta_{\alpha \beta}+\mathcal{T}_{\alpha \beta}\right)}{\partial x_{\beta}}=0
\end{aligned}
$$


supplemented by an entropy equation in non-conservative form for the energy transport

$$
\frac{\partial s}{\partial t}+u_{\alpha} \frac{\partial s}{\partial x_{\alpha}}=\frac{1}{\rho T}\left[\mathcal{T}_{\alpha \beta} \frac{\partial u_{\alpha}}{\partial x_{\beta}}-\frac{\partial q_{\beta}}{\partial x_{\beta}}\right]
$$

where the viscous tensor reads

$$
\mathcal{T}_{\alpha \beta}=\mu\left(\frac{\partial u_{\alpha}}{\partial x_{\beta}}+\frac{\partial u_{\beta}}{\partial x_{\alpha}}-\delta_{\alpha \beta} \frac{2}{3} \frac{\partial u_{\gamma}}{\partial x_{\gamma}}\right)
$$

with $\mu$ as dynamic viscosity, and the heat flux

$$
q_{\beta}=-\lambda \frac{\partial T}{\partial x_{\beta}}
$$

where $\lambda$ is the heat conductivity. The entropy field is connected to the flow fields through the ideal gas equation of state (EOS)

$$
p=\rho r T, s=C_{v} \ln \frac{p}{\rho^{\gamma}}
$$

with $C_{v}$ being the constant volume specific heat capacity, $r$ the specific gas constant and $\gamma \equiv C_{p} / C_{v}$ the adiabatic exponent.

In the original HRR- $p$ method $\sqrt[58]{ }$, the flow fields $(\rho$ and $\rho \boldsymbol{u}$ ) are computed by lattice Boltzmann method, while the entropy equation is updated using an explicit Euler method for time-integration supplemented by MUSCL scheme for advection along with a second order centered scheme for viscous terms. In practice, the density field evolves according to

$$
\begin{aligned}
\rho(\boldsymbol{x}, t+\Delta t) & =\rho-\frac{p}{c_{s}^{2}}+\sum_{i} f_{i}^{\mathrm{col}^{-}} \\
& =\rho-\sum_{i}\left[f_{i}^{\mathrm{col}}-f_{i}^{\mathrm{col}^{-}}\right]
\end{aligned}
$$

putting in evidence an identical mass flux $\left(f_{i}^{\mathrm{col}}-f_{i}^{\mathrm{col}^{-}}\right)$compared to classical density based LBMs. The momentum fields are updated as

$$
\left[\rho u_{\alpha}\right](\boldsymbol{x}, t+\Delta t)=\rho u_{\alpha}-\sum_{i} c_{i \alpha}\left(f_{i}^{\mathrm{col}}-f_{i}^{\mathrm{col}^{-}}\right)
$$

The collision populations $f_{i}^{\text {col }}$ are evaluated through a hybrid recursive regularized (HRR) collision procedure, yielding

$$
f_{i}^{\mathrm{col}}=f_{i}^{\mathrm{eq}}+\left(1-\frac{\Delta t}{\tau}\right) f_{i}^{\mathrm{neq}}+\frac{\Delta t}{2} F_{i}^{E}
$$


where the equilibrium part $\left(f_{i}^{\text {eq }}\right)$, the non-equilibrium part $\left(f_{i}^{\text {neq }}\right)$ and the forcing terms for viscous tensor $\left(F_{i}^{E}\right)$ are evaluated from their projections onto the D3Q19 rotational symmetry basis of Gauss-Hermite polynomials up to third order ${ }^{59}$,

$$
\begin{aligned}
& f_{i}^{\text {eq }}=\omega_{i}\left[\frac{p}{c_{s}^{2}}+\frac{\mathcal{H}_{i \alpha}^{(1)}}{c_{s}^{2}} \rho u_{\alpha}+\frac{\mathcal{H}_{i \alpha \beta}^{(2)}}{2 c_{s}^{4}} \rho u_{\alpha} u_{\beta}+\frac{\mathcal{H}_{i \gamma}^{(3 r)}}{6 c_{s}^{6}} a_{\gamma}^{(3 r), \text { eq }}\right] \\
& f_{i}^{\text {neq }}=\omega_{i}\left[\frac{\mathcal{H}_{i \alpha \beta}^{(2)}}{2 c_{s}^{4}} a_{\alpha \beta}^{(2) \text { neq }}+\frac{\mathcal{H}_{i \gamma}^{(3 r)}}{6 c_{s}^{6}} a_{\gamma}^{(3 r), \text { neq }}\right] \\
& F_{i}^{E}=\omega_{i} \frac{\mathcal{H}_{i \alpha \beta}^{(2)}}{2 c_{s}^{4}} a_{\alpha \beta}^{(2), E}
\end{aligned}
$$

where $\omega_{i}$ is the Gaussian weight associated to the discrete velocity $\boldsymbol{c}_{i}, p$ the pressure, and $c_{s} \equiv \Delta x /(\sqrt{3} \Delta t)$ the lattice speed of sound. Further details about this collision operation can be found in Appendix $\mathrm{D}$. The role of forcing term $F_{i}^{E}$ is to enforce conservativity (in the sense given in Refs. ${ }^{25126}$ ) by balancing errors coming from both truncation of the polynomial expansion of the polynomial expansion of the Maxwellian and the quadrature error associated to the lattice.

The objective of the current work is to couple the conservative form energy equation with the LBM via this hybrid approach. To this end, instead of using the non-conservative formulation of entropy equation (21), the conservative form of total energy or entropy equations are discretized with the proposed numerical schemes, i.e.

$$
\begin{aligned}
& \frac{\partial \rho E}{\partial t}+\frac{\partial \rho H u_{\alpha}}{\partial x_{\alpha}}=\frac{\partial \mathcal{T}_{\alpha \beta} u_{\alpha}}{\partial x_{\beta}}-\frac{\partial q_{\beta}}{\partial x_{\beta}} \\
& \frac{\partial \rho s}{\partial t}+\frac{\partial \rho s u_{\alpha}}{\partial x_{\alpha}}=\frac{1}{T}\left[\mathcal{T}_{\alpha \beta} \frac{\partial u_{\alpha}}{\partial x_{\beta}}-\frac{\partial q_{\beta}}{\partial x_{\beta}}\right]
\end{aligned}
$$

where $H \equiv E+p / \rho=e+\frac{1}{2} u_{\alpha} u_{\alpha}+p / \rho$ is the total enthalpy, and $e=C_{v} T$ the inner energy for idea gas. It is worth noting that when the scalar transport schemes are applied to the total energy equation, the scalar transported should be total enthalpy $(H)$ instead of total energy $(E)$. In the current work, the viscous terms in the R.H.S of Eq. (29) are resolved by second order central FD schemes same as in our previous works $\frac{5860}{5}$.

Using conservative formulation in energy transport should lead to conservation of the energy scalar. This is numerically guaranteed at the discrete level by the proposed schemes , i.e. the sum of all the scalar fluxes over any periodic domain is zero: 


$$
\begin{aligned}
\sum_{\boldsymbol{x}} \nabla^{U} \cdot(\rho \boldsymbol{u} \phi) & \equiv \sum_{\boldsymbol{x}}\left(\frac{1}{\Delta t} \sum_{i}\left[f_{i}^{\mathrm{col} \phi}-f_{i}^{\mathrm{col}-} \phi^{-}\right]\right)=0 . \\
\sum_{\boldsymbol{x}} \nabla^{C} \cdot(\rho \boldsymbol{u} \phi) & \equiv \sum_{\boldsymbol{x}}\left(\frac{1}{\Delta t} \sum_{i}\left[f_{i}^{\mathrm{col}} \frac{\phi^{+}+\phi}{2}-f_{i}^{\mathrm{col}-} \frac{\phi+\phi^{-}}{2}\right]\right) \\
& =\frac{1}{2 \Delta t}\left(\sum_{i} \sum_{\boldsymbol{x}}\left[f_{i}^{\mathrm{col}} \phi^{+}-f_{i}^{\mathrm{col}-} \phi\right]+\sum_{i} \sum_{\boldsymbol{x}}\left[f_{i}^{\mathrm{col}} \phi-f_{i}^{\mathrm{col}-} \phi^{-}\right]\right) \\
& =0 .
\end{aligned}
$$

The major benefit of the proposed schemes is the compatibility between the mass flux evaluation in the scalar transport equation and the one resolved by LBM. This feature is highlighted in the centered scheme $\nabla^{C}$, granting it a skew-symmetry property, since it also conserves the associated quadratic invariant $\rho \phi^{2}$ (see $e^{61}$ for a discussion about quadratic invariant conservation by numerical schemes). This can be demonstrated by examining the semi-discrete form of the scalar equation:

$$
\begin{aligned}
\sum_{\boldsymbol{x}} \delta_{t}\left(\rho \phi^{2}\right) & \equiv \sum_{\boldsymbol{x}}\left(2 \phi \delta_{t}(\rho \phi)-\phi^{2} \delta_{t} \rho\right) \\
& \equiv-\frac{1}{\Delta t} \sum_{\boldsymbol{x}}\left(2 \phi \nabla^{C} \cdot(\rho \boldsymbol{u} \phi)-\phi^{2} \sum_{i}\left[f_{i}^{\mathrm{col}}-f_{i}^{\mathrm{col}-}\right]\right) \\
& =-\frac{1}{\Delta t} \sum_{\boldsymbol{x}}\left(\phi \sum_{i}\left[f_{i}^{\mathrm{col}} \phi^{+}-f_{i}^{\mathrm{col}-} \phi+f_{i}^{\mathrm{col}} \phi-f_{i}^{\mathrm{col}-} \phi^{-}\right]-\phi^{2} \sum_{i}\left[f_{i}^{\mathrm{col}}-f_{i}^{\mathrm{col}-}\right]\right) \\
& =-\frac{1}{\Delta t} \sum_{\boldsymbol{x}} \sum_{i}\left(\phi f_{i}^{\mathrm{col}} \phi^{+}-\phi f_{i}^{\mathrm{col}-} \phi^{-}\right) \\
& =-\frac{1}{\Delta t} \sum_{i} \sum_{\boldsymbol{x}}\left(\phi^{+}\left(\phi f_{i}^{\mathrm{col}}\right)-\phi\left(\phi^{-} f_{i}^{\mathrm{col}-}\right)\right) \\
& =0
\end{aligned}
$$

Here the $\delta_{t}$ denotes the semi-discretized time derivation operator which only consider the convective part of the scalar evolution 61 . It is clear that invariant conservation is due to the identical mass flux evaluations in both the scalar flux and those resolved by LBM in Eq. (25). To the best of the authors' knowledge, none of the scalar transport schemes in the literature has such feature. 


\section{GLOBAL ACCURACY OF THE HYBRID LATTICE BOLTZMANN METHOD}

Although the analysis based on Taylor expansion displayed in Appendix A gives the order of accuracy of the proposed spatial operators, the global order of segregated solver should be studied using numerical test cases. To this end, an one-dimensional entropy spot advection is considered ${ }^{58}$. This test case is initialized with an uniform initial velocity field corresponding to $u=1$ (approximately at Ma 0.2 ) on a periodic one-dimensional domain of $L=1$. Other variables are initialized according to

$$
\begin{aligned}
& \rho=\rho_{0}\left[1+\delta \exp \frac{-\left(x-x_{0}\right)^{2}}{R^{2}}\right] \\
& T=T_{0}\left[1-\delta \exp \frac{-\left(x-x_{0}\right)^{2}}{R^{2}}\right]
\end{aligned}
$$

In practice, the parameters are chosen as $T_{0}=20, \rho_{0}=1, x_{0}=0.5, R=0.05$ and $\delta=0.001$. The time step is given such that the CFL number is around 0.5. The energy scalar transported in the current test is the total energy following Eqn. 29a), without any viscous term to mimic a Euler calculation. Flow fields after one flow-over time are compared to their reference solutions (which are their initial values for this specific test). The global order of the solver is studied by the relative error defined as

$$
\epsilon(\phi) \equiv \sqrt{\frac{\int\left(\phi-\phi_{\text {ref }}\right)^{2} d x}{\int \phi_{\text {ref }}^{2} d x}}
$$

Relative errors for density, velocity and energy are plotted versus the number of points in the calculation domain as figure 1. It is quite clear that the global convergence rate of the hybrid LBM solver with the total energy equation discretized by the upwinded operator $\nabla^{U}$ achieves first order, and the global order of the center operator $\nabla^{C}$ simulation is around two. Note that the flat tails in Fig. 1(b) merely indicate that the velocity fields reach the range of the machine tolerance with the chosen grid.

\section{TEST CASE: COMPRESSIBLE TAYLOR-GREEN VORTEX}

The inviscid Taylor-Green vortex (TGV) test-case is addressed to assess the conservation properties of the proposed numerical schemes. It is calculated in a $2 \pi \times 2 \pi \times 2 \pi$ periodic domain with only 32 grid points in each direction to emphasize the stability feature of the 


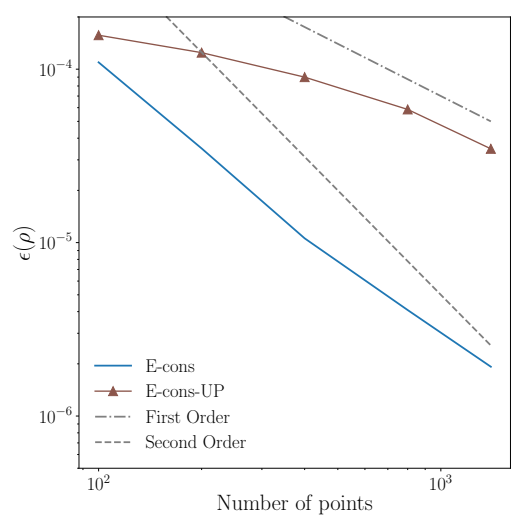

(a)

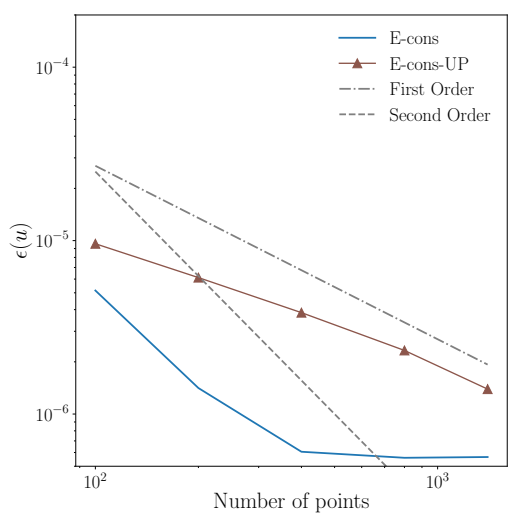

(b)

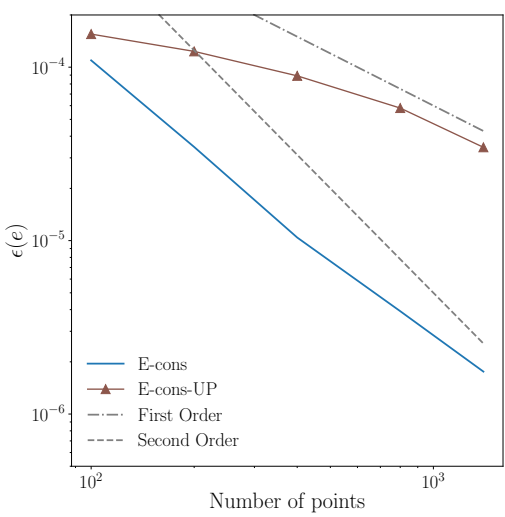

(c)

Figure 1: Error on density (a), velocity (b) and internal energy (c) as a function of the spatial discretization for the numerical schemes $\nabla^{C}$ (E-cons) and $\nabla^{U}$ (E-cons-UP), for the entropy spot convection test case.

schemes. The viscosity is set to $10^{-25} \mathrm{~kg} / \mathrm{m} / \mathrm{s}$ to mimic an inviscid condition for LBM solvers 23 . The initial fields are given by Eqn (35), corresponding to constant density initial conditions (CDIC) at given Ma numbers 6263 .

$$
\begin{aligned}
& u_{x}=\sin x \cos y \cos z \\
& u_{y}=-\cos x \sin y \cos z \\
& u_{z}=0 \\
& \rho=1 \\
& p=\frac{1}{\gamma \mathrm{Ma}^{2}}+\frac{1}{16}(\cos 2 x+\cos 2 y)(\cos 2 z+2)
\end{aligned}
$$

Numerical configurations tested in the current work are listed in Table If. The density and momentum fields are all resolved by the HRR- $p$ LBM flow solver ${ }^{[58}$ while the energy scalar transport is modeled using several different solutions: internal energy, total energy and entropy are used in either conservative or primitive form. Only the Euler part of the 
energy transport equations is considered to emphasize the impact of the flux schemes, i.e.

$$
\begin{aligned}
& \frac{\partial \rho E}{\partial t}+\frac{\partial \rho H u_{\alpha}}{\partial x_{\alpha}}=0 \\
& \frac{\partial e}{\partial t}+u_{\alpha} \frac{\partial e}{\partial x_{\alpha}}+\frac{p}{\rho} u_{\alpha, \alpha}=0 \\
& \frac{\partial \rho s}{\partial t}+\frac{\partial \rho s u_{\alpha}}{\partial x_{\alpha}}=0 \\
& \frac{\partial s}{\partial t}+u_{\alpha} \frac{\partial s}{\partial x_{\alpha}}=0
\end{aligned}
$$

The second order accurate isotropic spatial derivation operator used in $\underline{60}$ or MUSCL schemes employed in $\frac{58}{5}$ are applied for the energy scalar gradients in primitive form. The scalar flux in conservative form are discretized using either the $\nabla^{C}$ and $\nabla^{U}$ operators, or the low-dissipation scalar transport scheme proposed in ${ }^{53}$ in its conservative form given by

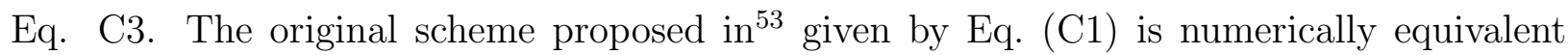
to our $\nabla^{U}$ operator, therefore it is not tested separately. Also, although the operator $\nabla^{O S}$

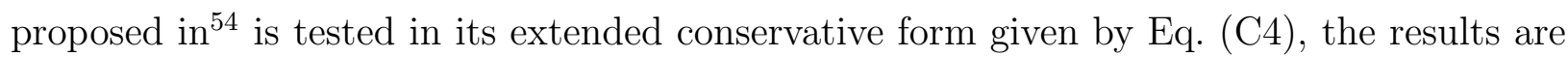
not reported in the current work because they are very close to those obtained by operator $\nabla^{U}$. The time integration of the scalar equation is performed using a first-order explicit Euler scheme to achieve a two-way coupling between energy scalar solvers and the LBM flow solver ${ }^{64}$, with an acoustic CFL number close to 0.5 .

The variables that are conserved numerically are listed in Table III. It is worth noting that the HRR-p LBM flow solver always conserves density and momentum, as proved in Appendix E. As demonstrated in Eq. (31), the case $s$ - cons should conserve both the linear invariant $(\rho s)$ and the quadratic invariant $\rho s^{2}$. However, in the case $E-$ cons, $\rho E^{2}$ is only globally conserved due to presence of the pressure work $p u_{\alpha}$ in the flux other than only $\rho E u_{\alpha}$, as pointed out in ${ }^{61}$. Two distinct Ma numbers, Ma $=0.08$ and $\mathrm{Ma}=0.8$, are chosen to cover both nearly incompressible and compressible regimes.

\section{A. Nearly incompressible case}

The temporal evolution of the concerned quantities when Mach number is 0.08 are displayed in Fig. 2. The results are reported by the normalized displacement from their original 
Table I: Nomenclature in Taylor-Green vortex simulations

\begin{tabular}{|c|c|c|c|}
\hline Configuration & ergy scalar & equ & Flux scheme \\
\hline E-cons & Equation & $(36 a)$ & Equation 15 \\
\hline e-prim & Equation & $(36 \mathrm{~b})$ & $2^{\text {ed }}$ order isotropic 60 \\
\hline s-cons & Equation & $(36 c)$ & Equation (15) \\
\hline s-prim & Equation & $(36 \mathrm{~d})$ & $2^{\text {ed }}$ order isotropic 60 \\
\hline s-prim-MU & Equation & $(36 \mathrm{~d})$ & MUSCL 58 \\
\hline E-cons-UP & Equation & (36a) & Equation (14) \\
\hline E-cons-Onishi & Equation & $(36 a)$ & Equation C3 53 \\
\hline s-cons-Onishi & Equation & $36 c$ & Equation \\
\hline
\end{tabular}

Table II: Variable conserved locally $\checkmark$ or globally $(\checkmark)$ by tested numerical schemes

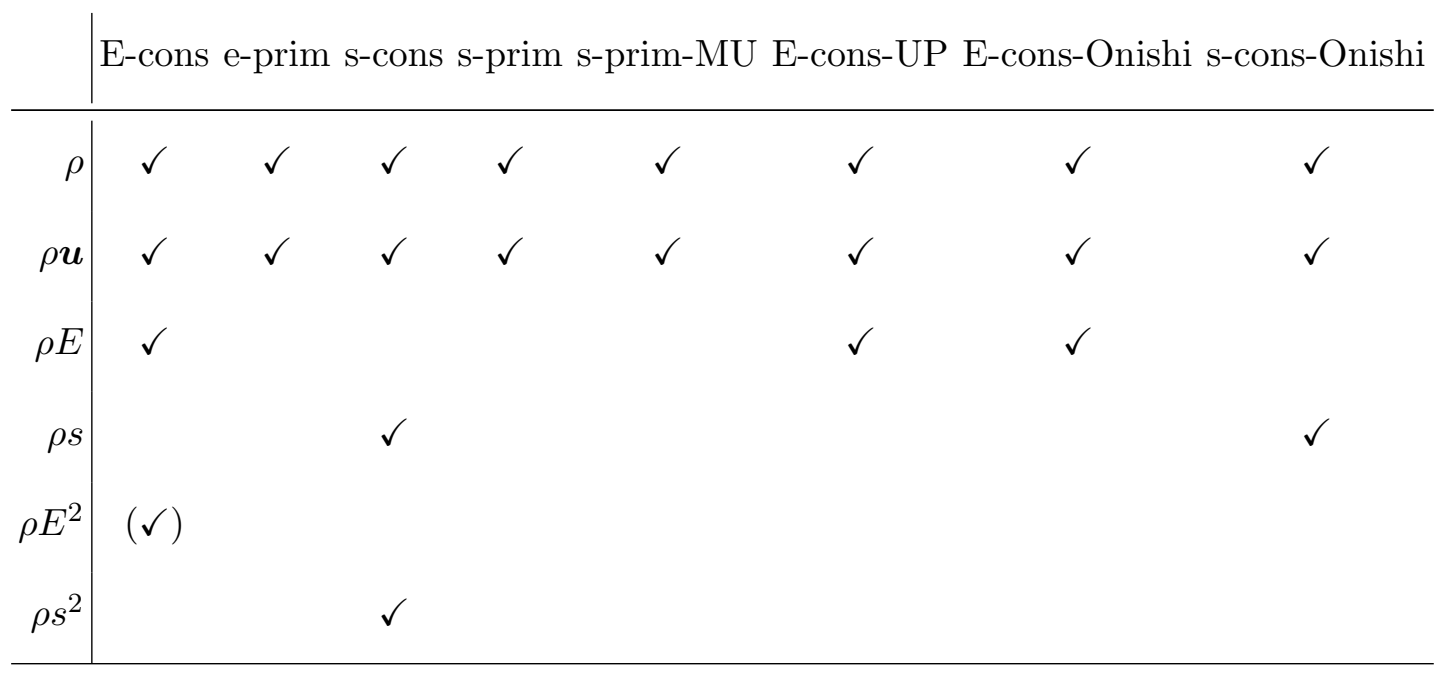

values $^{61}$, i.e.

$$
\langle\bar{f}\rangle \equiv \frac{\bar{f}-\overline{f_{0}}}{\left|\overline{f_{0}}\right|} \equiv \frac{\int f(t) d \mathcal{V}-\int f(0) d \mathcal{V}}{\left|\int f(0) d \mathcal{V}\right|}
$$

All numerical configurations succeed in conducting this test up to $t=10$ except Econs-Onishi and s-cons-Onishi, where the low-dissipation operators $\nabla^{O C}$ proposed in ${ }^{53}$ is 
employed. The simulations s-cons-Onishi becomes unstable almost immediately after the sinitial time and therefore the results are not plotted. The simulation E-cons-Onishi remains stable up to $t \simeq 1$. This is not surprising because it can be shown from a Taylor analysis of the operator $\nabla^{O C}$ that, its numerical dissipation is proportional to $\rho \boldsymbol{u u}$, which is too small to stabilize the simulation at this Mach number. It is seen from Fig. 2 that in this low Mach configuration, the density and momentum are conserved numerically in all numerical recipes at a tolerance of $10^{-10}$. The kinetic energy from different schemes are decaying at almost the same rate as shown in Fig. 2(c), which is reasonable for hybrid LBM solvers because at low Mach number, the variation of kinetic energy should be dominated by the dissipation properties of the LBM flow solver which is identical in all setups. The kinetic energy maintains approximately $70 \%$ of its original value at $t=7$, which is even better than the performance of some high-order compressible schemes on much finer grids ${ }^{62}$.

Scalar fields listed in table II are all conserved as expected, which can be seen in a zoomed view of the corresponding curves in Figure 3. The entropy quadrature $\left\langle\rho s^{2}\right\rangle$ in the s-cons configuration plotted in Figure 3(c) conserves with a tolerance of $10^{-9}$, which proves that the scalar operator $\nabla^{C}$ in equation 15 is quadrature preserving. The total energy quadrature $\left\langle\rho E^{2}\right\rangle$ resolved from configuration E-cons and E-cons-UP are given in Figure 3(d), Although this quantity is only globally conserved ${ }^{61}$, its variation is very small (at a tolerance of $10^{-6}$ ), because the efforts of the pressure work should be very small at low Mach number.

It is also worth noting that the primitive formulation of the entropy equation (Eq. $36 \mathrm{~d}$ ) equipped with an isotropic spacial operator (s-prim) performs quite well in preserving energy quantities under this nearly incompressible case. It effectively preserves the entropy ( $\rho s)$ and the associated quadratic invariant $\rho s^{2}$. Even the internal energy and its quadratic invariant $\left(\rho e, \rho e^{2}\right)$ are nearly conserved as seen in Fig. 3. The primitive formulation of entropy with

MUSCL scheme (s-prim-MU) applied in our previous works ${ }^{23158165}$ for compressible flows fails to conserve any energy scalar due to the complex numerical treatment involving much larger stencils, but it gives a stable solution.

\section{B. Higher Mach number case}

The compressible simulations of TGV are performed at Ma=0.8. At this Mach number, operator $\nabla^{O C}$ leads to stable simulations. The conservative variables are displayed in Fig. 4 


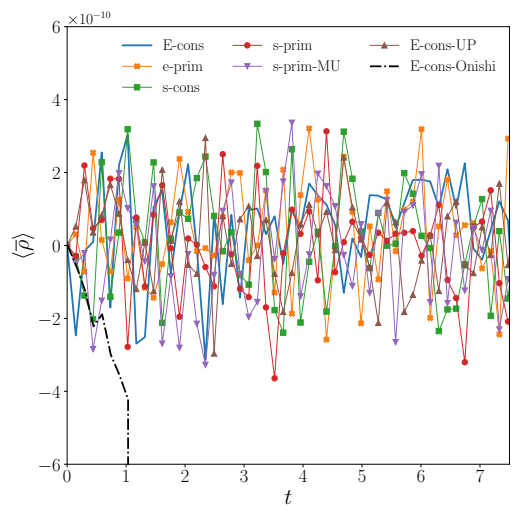

(a)

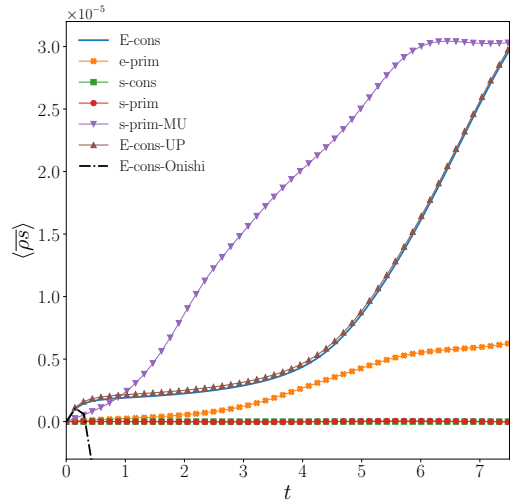

(d)

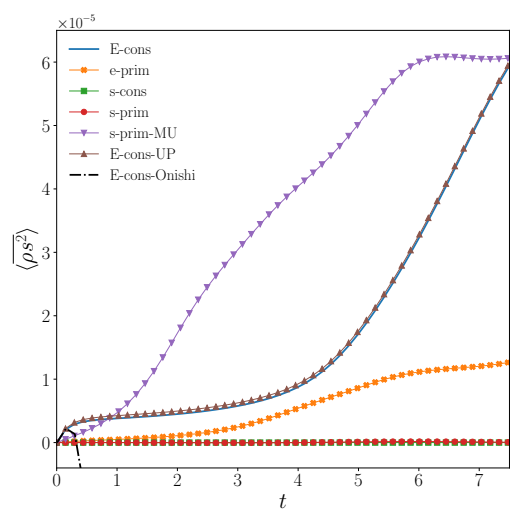

(g)

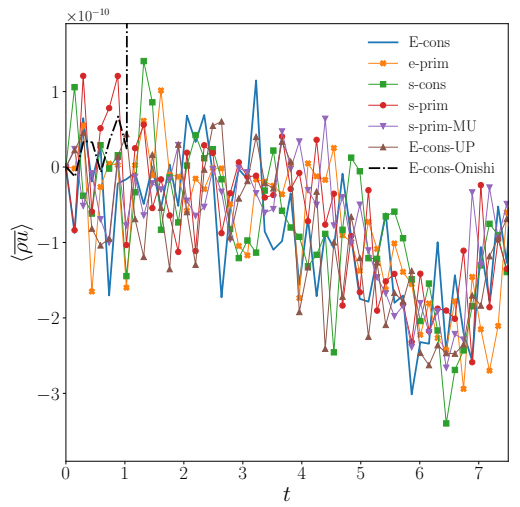

(b)

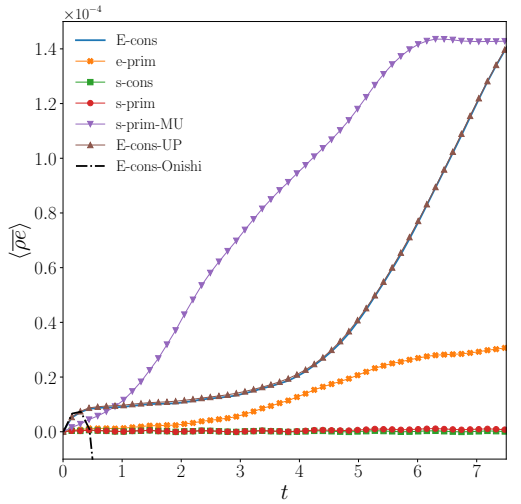

(e)

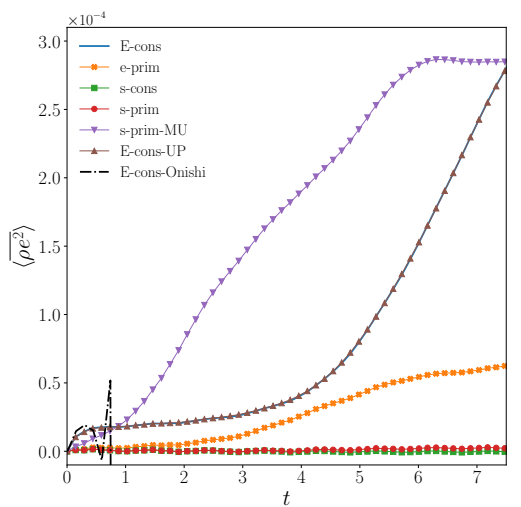

(h)

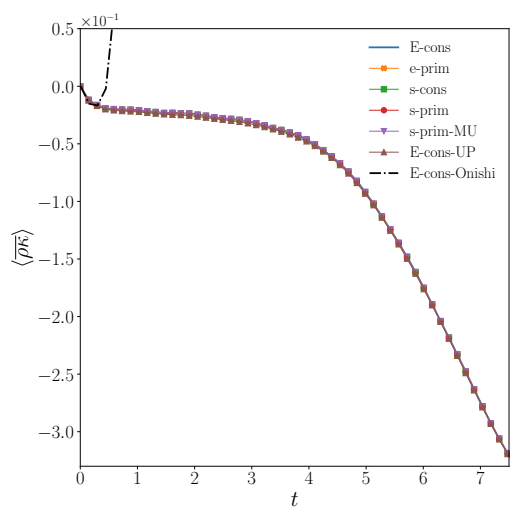

(c)

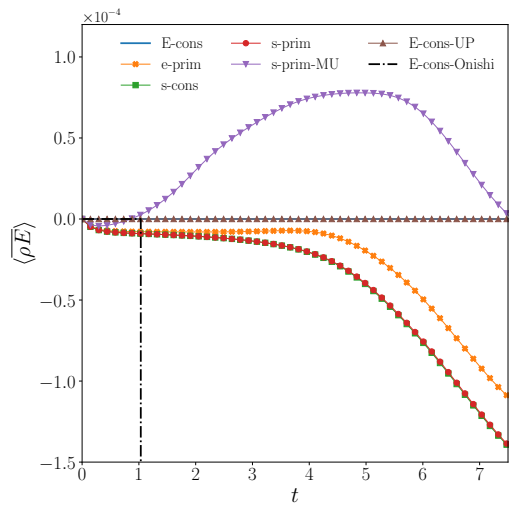

(f)

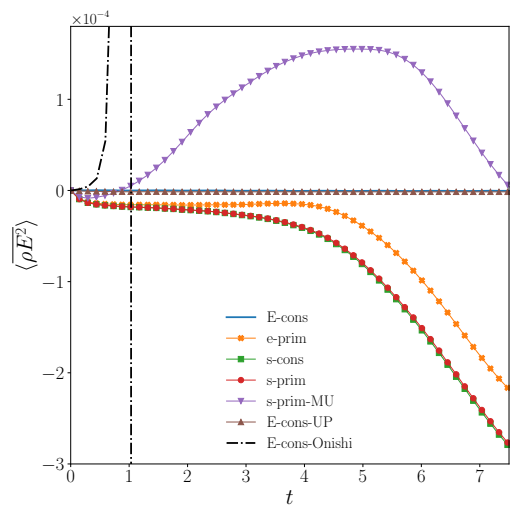

(i)

Figure 2: Temporal evolution of (a) $\rho$, (b) $\rho u$, (c) $\rho \kappa$, (d) $\rho s$, (e) $\rho e$, (f) $\rho E$, (g) $\rho s^{2}$, (h) $\rho e^{2}$, (i) $\rho E^{2}$ quantities in TGV simulation from different configurations at $\mathrm{Ma}=0.08$. 


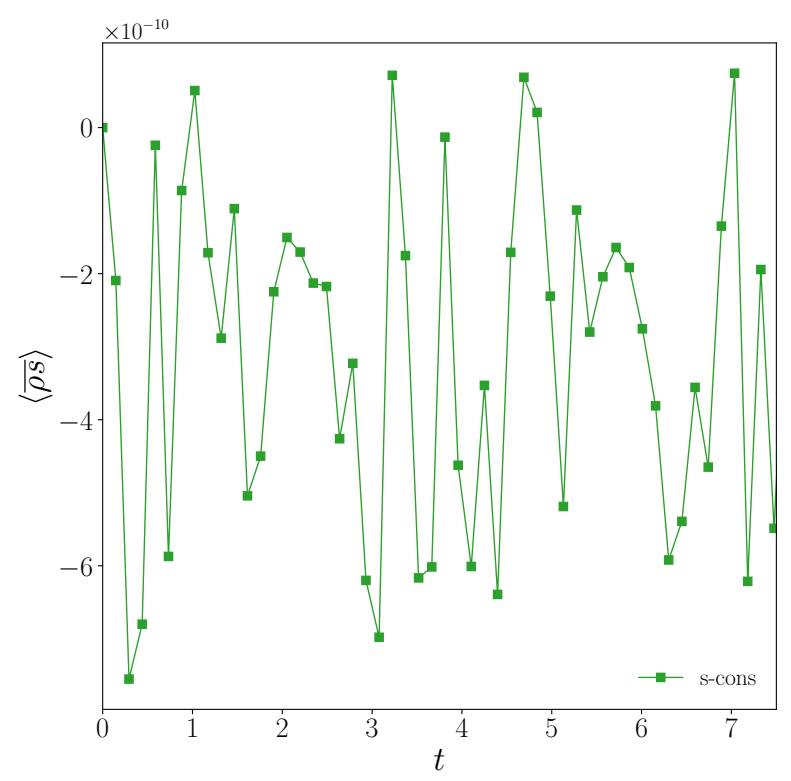

(a)

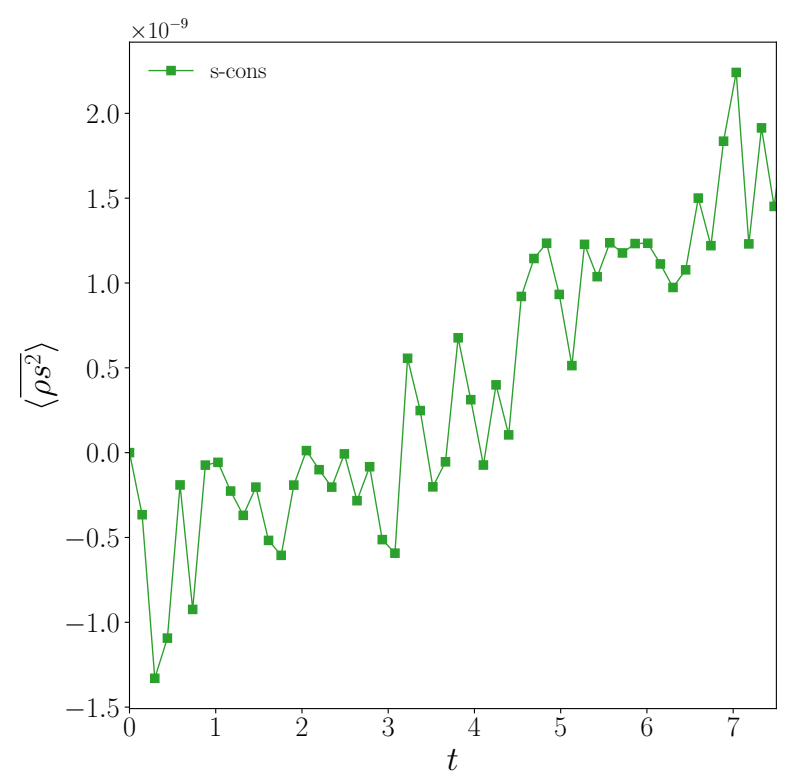

(c)

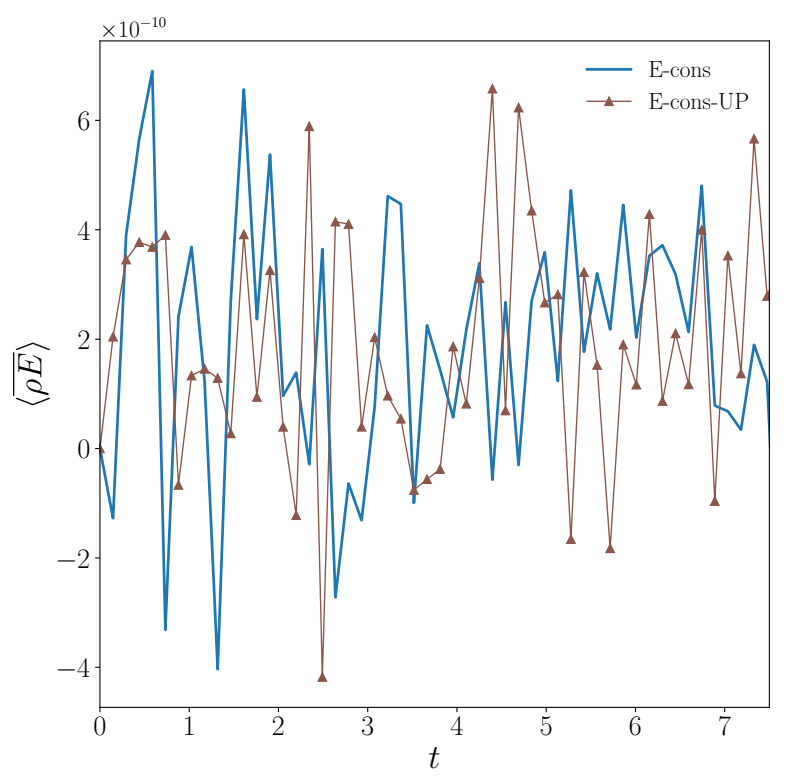

(b)

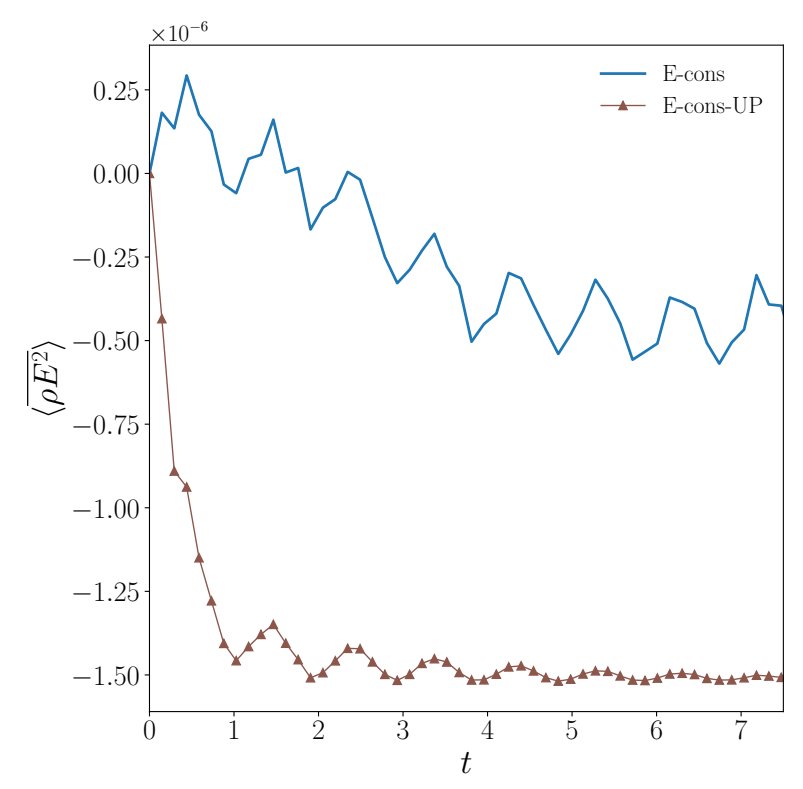

(d)

Figure 3: Temporal evolution of conserved scalars (a) $\rho s$, (b) $\rho E$, (c) $\rho s^{2}$, (d) $\rho E^{2}$ in TGV test cases at $\mathrm{Ma}=0.08$.

using the ratio between their spatial integration at time $t$ and the initial values, i.e.

$$
\frac{\bar{f}}{\bar{f}_{0}} \equiv \frac{\int f(t) d \mathcal{V}}{\int f(0) d \mathcal{V}}
$$


The conservative properties of the schemes are the same as in Table II, although the total energy quadratic invariant $\rho E^{2}$ which is globally conserved in E-cons configuration varies significantly because of the non-negligible pressure work effects at this higher Mach number. Nevertheless, the proposed schemes are still stable on this coarse grid, and the conservation properties are still observed.

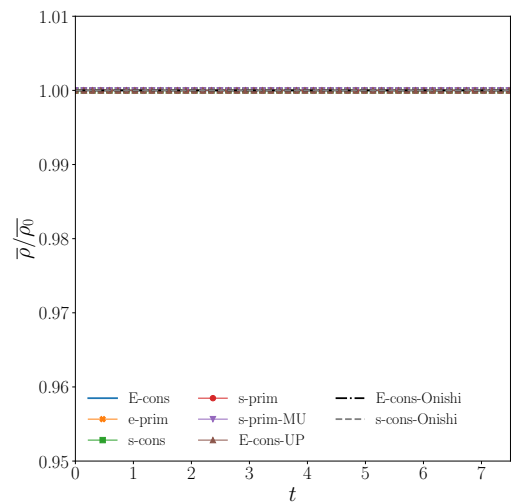

(a)

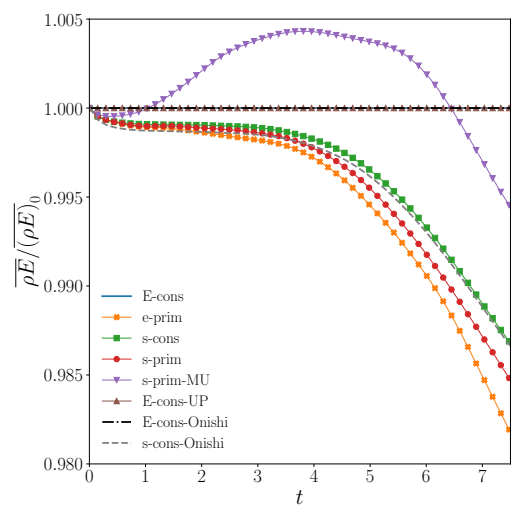

(d)

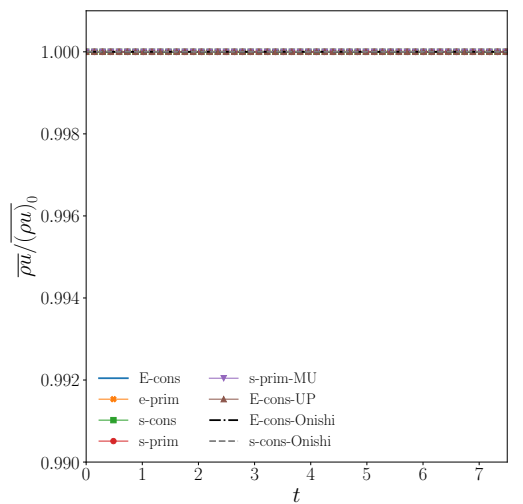

(b)

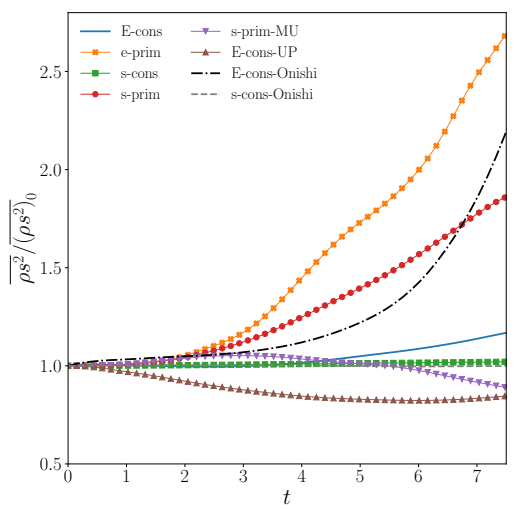

(e)

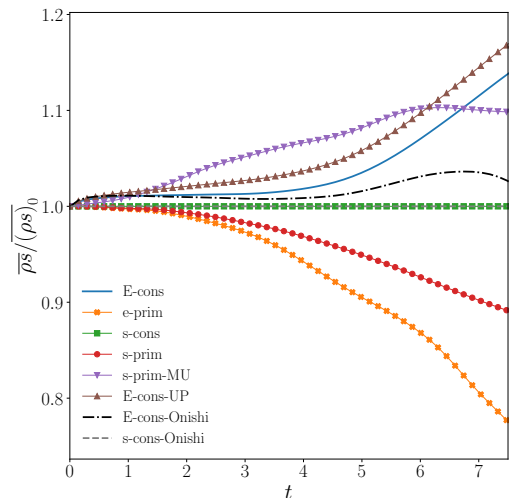

(c)

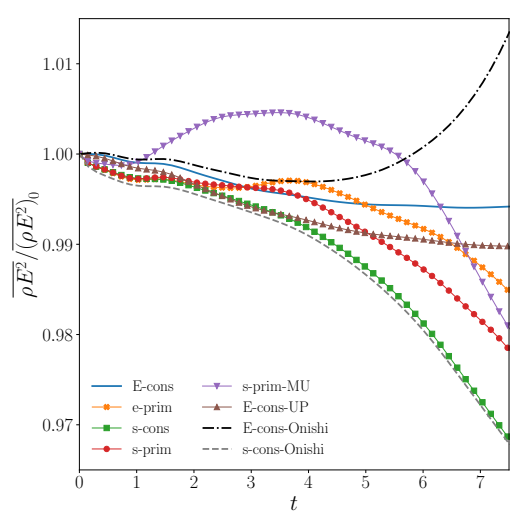

(f)

Figure 4: Temporal evolution of statistical (a) $\rho$, (b) $\rho u$, (c) $\rho s$, (d) $\rho E$, (e) $\rho s^{2}$, (f) $\rho E^{2}$ quantities in TGV simulation from different configurations, $\mathrm{Ma}=0.8$.

\section{COMPRESSIBLE CASES WITH DISCONTINUITIES}

Having confirmed the stability and conservative properties of the proposed schemes, we now assess their performances in the presence of shock waves. To deal with discontinuities, we combine the schemes given in Eqs. (14) and (15) dynamically using a shock sensor already 
used in our previous works 23158 . When a discontinuity is detected by the sensor, the fully upwind operator $\nabla^{U}$ given by Eq. (14) is applied, while the centered scheme $\nabla^{C}$ defined in Eq. (15) is applied elsewhere. Artificial viscosity obtained by the Jameson shock sensor ${ }^{66}$ is

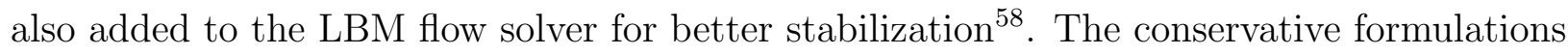
of total energy and entropy are tested with the dynamic upwinding, denoted by E-consdyn and s-cons-dyn in the following. A one-dimensional shock-tube and a two-dimensional Riemann configuration are simulated.

\section{A. SOD shock-tube case}

This classical one-dimensional Sod shock tube case is set initially as $\left(\rho_{L}, u_{L}, p_{L}\right)=(1,0,1)$ and $\left(\rho_{R}, u_{R}, p_{R}\right)=(0.125,0,0.1)$. The computational domain is discretized by 400 points with an acoustic CFL number around 0.5. The density, velocity and pressure fields at $t=0.2$ are shown in Fig. 5. Apart from the numerical methods E-cons-dyn and s-cons-dyn

proposed in the current work, the s-prim-MU scheme ${ }^{58}$ mentioned in the TGV test case is also examined. A reference solution obtained from an Riemann solver on a much finer grid (2000 points) is also provided. It can be seen that all numerical configurations lead to stable solutions at $t=0.2$, however, only the E-cons-dyn scheme gives the correct inner energy profile. This is due to the fact that, under discrete form, only the conservative total energy equation can lead to the appropriate Hugoniot relations through a strong discontinuity 67.

\section{B. Two-dimensional Riemann problems}

Two-dimensional Riemann problems proposed in ${ }^{68}$ are considered to investigate the multidimensional performance of the proposed schemes. Configurations No. 3, No. 4, No. 6 and No. 12 in $\frac{68}{6}$ are selected. All these simulations are stable using E-cons-dyn and s-cons-dyn schemes with a grid revolution of $400 \times 400$, but as for the SOD test case, only total energy equation leads to correct solutions when strong shocks are encountered. Figure 6 displays the density iso-contours of configuration No.3 resolved by different schemes. The initial condition of this configuration is given in Table III. Compared to the reference solutions reported in $\frac{68}{6}$, only the E-cons-dyn case recovers the correct $2 \mathrm{D}$ pattern. 


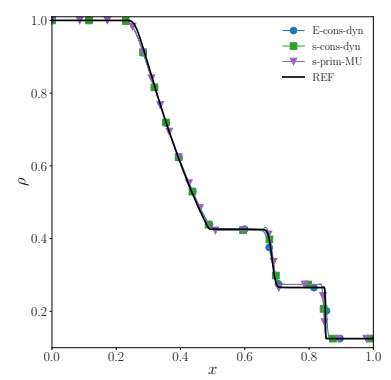

(a)

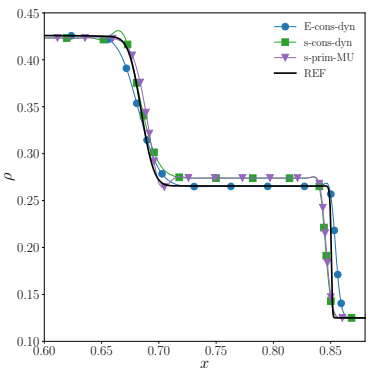

(e)

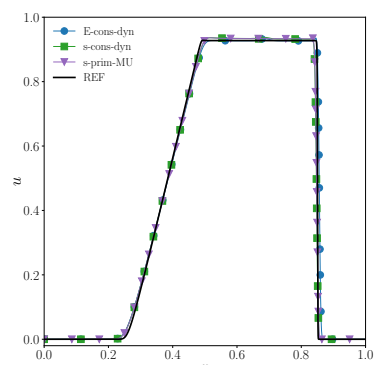

(b)

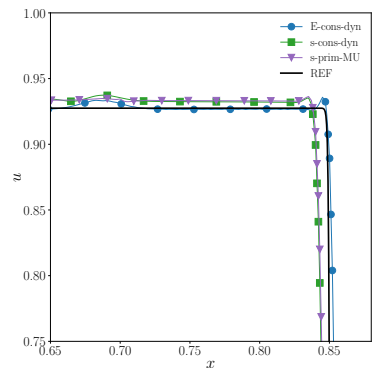

(f)

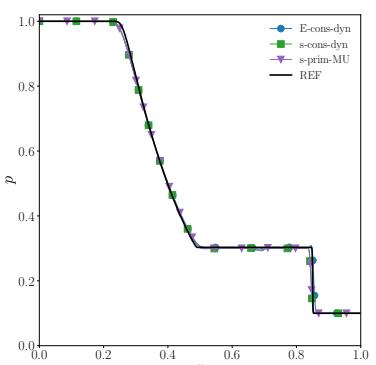

(c)

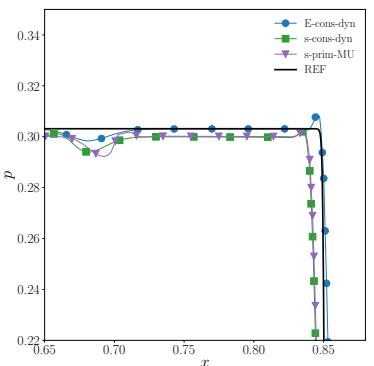

$(\mathrm{g})$

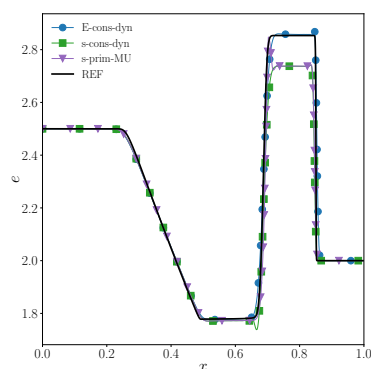

(d)

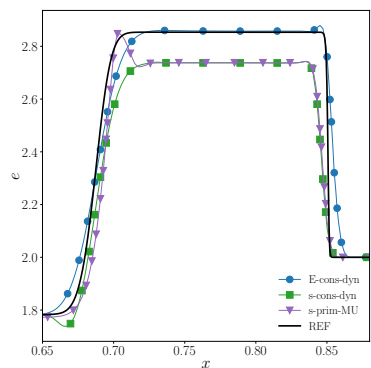

(h)

Figure 5: Density, velocity, pressure and internal energy evolution (resp. a, b, c, d) in SOD shock tube at $t=0.2$ resolved by different schemes. (e, f, g, h) correspond to the same plots, zoomed in the region of interest.

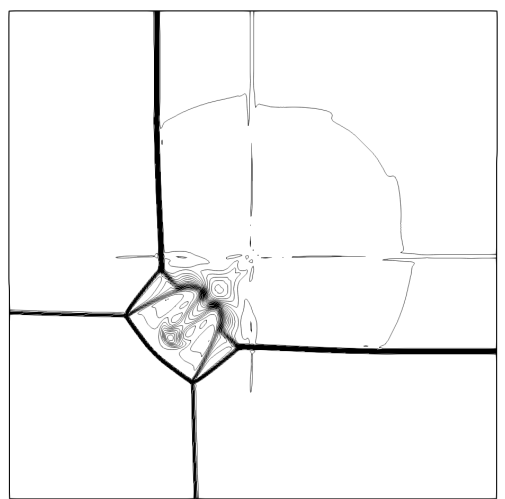

(a)

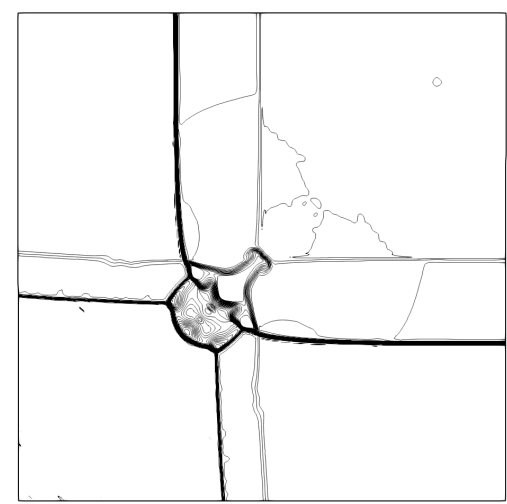

(b)

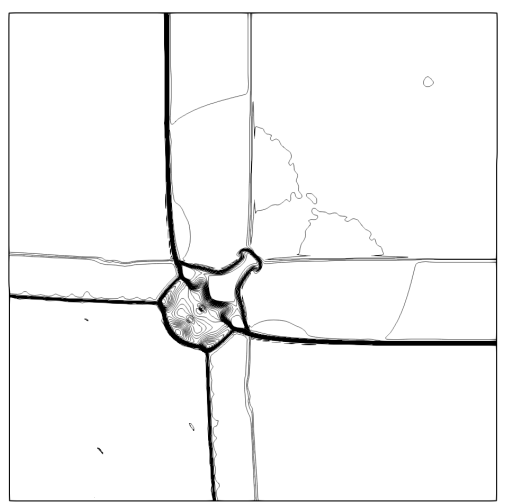

(c)

Figure 6: Density iso-contours of 2D Riemann case No. 3 in $\frac{68}{6}$ at $t=0.3$ obtained with (a) E-cons-dyn, (b) s-cons-dyn and (c) s-prim-MU schemes. 


$$
\begin{array}{ll|ll}
\rho=0.5323 & p=0.3 & \rho=1.5 & p=1.5 \\
u=1.206 & v=0 & u=0 & v=0 \\
\hline \rho=0.138 & p=0.029 & \rho=0.5323 & p=0.3 \\
u=1.206 & v=1.206 & u=0 & v=1.206
\end{array}
$$

Table III: Riemann 2D conf. $3^{68}$

\section{CONCLUSIONS AND DISCUSSIONS}

We have presented a new numerical scheme for use in segregated hybrid LB models, i.e. models consisting of a LB solver for mass and momentum conservation coupled with a FD solver for the macroscopic energy conservation equation (or another scalar). The numerical scheme was specifically designed to remove the inconsistency between mass conservation treated by the LB solver, on one hand, and the mass conservation hidden into the scalar equation (e.g. total energy) in conservative form.

This is particularly useful for compressible flows, where the equivalence between the various forms of energy equation is only valid in continuous form. When discontinuities are encountered, only the total energy equation in conservative form can lead to the correct Hugoniot jump relations. The ability to accurately jump conditions was shown on challenging one and two-dimensional Riemann problems.

The result, applied to the HRR- $p$ model proposed ${ }^{58}$, is a numerical method that not only numerically conserves $\rho, \rho \boldsymbol{u}$, but also $\rho E$ (and $\rho E^{2}$ globally), a characteristic which was never obtained, to the authors knowledge, using a hybrid LBM formulation.

Compared with the MUSCL scheme used in our previous work ${ }^{58}$, another advantage besides leading to adequate jump conditions - is that it only requires the nearest-neighbor information, thus reducing MPI exchanges in an HPC framework.

The scheme can also be adapted easily to take into account external forces work, e.g. gravity, by simply modifying the flux to be computed ( $\rho H$ in the case of total energy - see Eq. (29a).

Lastly, its simple implementation allows to dynamically include upwinding where required 
to enhance stability (at the cost of additional numerical diffusion).

\section{Acknowledgements}

The French Space agency (CNES) is acknowledged for supporting Song Zhao at M2P2. Centre de Calcul Intensif d'Aix-Marseille and GENCI-TGCC/CINES (Grant 2018-A0032A07679) are acknowledged for granting access to their high performance computing resources. We also acknowledge support from Labex MEC (ANR-10-LABX-0092) and the A*MIDEX project (ANR-11-IDEX-0001-02), funded by the "Investissements d'Avenir" and ANR Industrial Chair ALBUMS (grant ANR-18-CHIN-0003-01). The work was performed using the ProLB solver.

\section{DATA AVAILABILITY}

The data that support the findings of this study are available from the corresponding author upon reasonable request.

\section{Appendix A: Second order accuracy of the LBM mass flux evaluation}

To demonstrate the order of accuracy of the mass flux evaluation in LBM, let first expand the momentum transport using equation (7)

$$
\left(\rho u_{\alpha}\right)(t+\Delta t, \boldsymbol{x})=\rho u_{\alpha}-\Delta t \frac{\partial}{\partial x_{\beta}} \Pi_{\alpha \beta}^{f^{\mathrm{col}}}+\mathcal{O}\left(\Delta t^{2}\right)
$$

This equation leads to an expression of the cross derivation of the second order moments of $f^{\mathrm{col}}$

$$
\begin{aligned}
\frac{\partial^{2}}{\partial x_{\alpha} x_{\beta}} \Pi_{\alpha \beta}^{f^{\mathrm{col}}} & =\frac{\partial}{\partial x_{\alpha}}\left(\frac{\partial}{\partial x_{\beta}} \Pi_{\alpha \beta}^{f^{\mathrm{col}}}\right) \\
& =\frac{1}{\Delta t} \frac{\partial}{\partial x_{\alpha}}\left[\rho u_{\alpha}-\left(\rho u_{\alpha}\right)(t+\Delta t, \boldsymbol{x})\right]+\mathcal{O}(\Delta t)
\end{aligned}
$$


Substituting the corresponding term in the the LBM mass flux scheme Eq. (9) will give a second order accuracy of the mass flux evaluation as in equation (A3)

$$
\begin{aligned}
\nabla^{L} \cdot(\rho \boldsymbol{u}) & =\frac{\partial}{\partial x_{\alpha}} \rho u_{\alpha}-\frac{\Delta t}{2} \frac{\partial^{2}}{\partial x_{\alpha} x_{\beta}} \Pi_{\alpha \beta}^{f^{\mathrm{col}}}+\mathcal{O}\left(\Delta t^{2}\right) \\
& =\frac{\partial}{\partial x_{\alpha}} \rho u_{\alpha}-\frac{\Delta t}{2}\left[\frac{1}{\Delta t} \frac{\partial}{\partial x_{\alpha}}\left[\rho u_{\alpha}-\left(\rho u_{\alpha}\right)(t+\Delta t, \boldsymbol{x})\right]+\mathcal{O}(\Delta t)\right] \\
& =\frac{\partial}{\partial x_{\alpha}} \frac{\rho u_{\alpha}+\left(\rho u_{\alpha}\right)(t+\Delta t, \boldsymbol{x})}{2}+\mathcal{O}\left(\Delta t^{2}\right)
\end{aligned}
$$

It is worth noting that although equation 10 is generally valid for any LBM distributions, there is one essential requirement to make the flux evaluation second order as indicated in the above deductions: the next order moments, e.g. mass flux $\rho u_{\alpha}$ here, must be also transported by the same population. In contract, the population $f^{\phi}$ introduced in Eq. 12 , only servers to transport the scalar field, the scalar flux $\rho u_{\alpha} \phi$ are not governed by the same population anymore. As a matter of fact, the velocity components in the scalar flux are updated by the population $f$ in practice. Thus, the upwinded divergence operator $\nabla^{U}$ can not achieve second order by time marching. 


\section{Appendix B: Taylor expansion of the center scalar operator}

$$
\begin{aligned}
& \nabla^{C} \cdot(\rho \boldsymbol{u} \phi) \equiv \frac{1}{2 \Delta t}\left[\phi\left(\Delta t \frac{\partial \rho u_{\alpha}}{\partial x_{\alpha}}-\frac{\Delta t^{2}}{2} \frac{\partial^{2} \Pi_{\alpha \beta}^{\mathrm{fol}}}{\partial x_{\alpha} \partial x_{\beta}}+\mathcal{O}\left(\Delta t^{3}\right)\right)\right. \\
& +\left(\rho \phi+\Delta t \rho u_{\alpha} \frac{\partial \phi}{\partial x_{\alpha}}+\frac{\Delta t^{2}}{2} \Pi_{\alpha \beta}^{f \text { col }} \frac{\partial^{2} \phi}{\partial x_{\alpha} \partial x_{\beta}}+\mathcal{O}\left(\Delta t^{3}\right)\right) \\
& \left.-\left(\rho \phi-\Delta t \frac{\partial \rho u_{\alpha} \phi}{\partial x_{\alpha}}+\frac{\Delta t^{2}}{2} \frac{\partial^{2} \phi \Pi_{\alpha \beta}^{f^{c o l}}}{\partial x_{\alpha} \partial x_{\beta}}+\mathcal{O}\left(\Delta t^{3}\right)\right)\right] \\
& =\frac{\partial}{\partial x_{\alpha}} \rho u_{\alpha} \phi+\frac{\Delta t}{4}\left[-\phi \frac{\partial^{2} \Pi_{\alpha \beta}^{f \mathrm{col}}}{\partial x_{\alpha} \partial x_{\beta}}+\Pi_{\alpha \beta}^{f \mathrm{col}} \frac{\partial^{2} \phi}{\partial x_{\alpha} \partial x_{\beta}}-\frac{\partial^{2} \phi \Pi_{\alpha \beta}^{f \mathrm{col}}}{\partial x_{\alpha} \partial x_{\beta}}\right]+\mathcal{O}\left(\Delta t^{2}\right) \\
& =\frac{\partial}{\partial x_{\alpha}} \rho u_{\alpha} \phi+\frac{\Delta t}{4}\left[-\phi \frac{\partial^{2} \Pi_{\alpha \beta}^{f^{\mathrm{col}}}}{\partial x_{\alpha} \partial x_{\beta}}+\Pi_{\alpha \beta}^{f^{\mathrm{col}}} \frac{\partial^{2} \phi}{\partial x_{\alpha} \partial x_{\beta}}-\frac{\partial}{\partial x_{\alpha}} \frac{\partial \phi \Pi_{\alpha \beta}^{f^{\mathrm{col}}}}{\partial x_{\beta}}\right]+\mathcal{O}\left(\Delta t^{2}\right) \\
& =\frac{\partial}{\partial x_{\alpha}} \rho u_{\alpha} \phi+\frac{\Delta t}{4}\left[-\phi \frac{\partial^{2} \Pi_{\alpha \beta}^{f^{\mathrm{col}}}}{\partial x_{\alpha} \partial x_{\beta}}+\Pi_{\alpha \beta}^{f^{\mathrm{col}}} \frac{\partial^{2} \phi}{\partial x_{\alpha} \partial x_{\beta}}\right. \\
& \left.-\frac{\partial}{\partial x_{\alpha}}\left(\phi \frac{\partial \Pi_{\alpha \beta}^{f^{\mathrm{col}}}}{\partial x_{\beta}}+\Pi_{\alpha \beta}^{f^{\mathrm{col}}} \frac{\partial \phi}{\partial x_{\beta}}\right)\right]+\mathcal{O}\left(\Delta t^{2}\right) \\
& =\frac{\partial}{\partial x_{\alpha}} \rho u_{\alpha} \phi+\frac{\Delta t}{4}\left[-\phi \frac{\partial^{2} \Pi_{\alpha \beta}^{f^{\mathrm{col}}}}{\partial x_{\alpha} \partial x_{\beta}}\right. \\
& \left.-\left(\frac{\partial \phi}{\partial x_{\alpha}} \frac{\partial \Pi_{\alpha \beta}^{f \circ o l}}{\partial x_{\beta}}\right)-\phi \frac{\partial^{2} \Pi_{\alpha \beta}^{f^{c o l}}}{\partial x_{\alpha} \partial x_{\beta}}-\left(\frac{\partial \Pi_{\alpha \beta}^{f^{c o l}}}{\partial x_{\alpha}} \frac{\partial \phi}{\partial x_{\beta}}\right)\right]+\mathcal{O}\left(\Delta t^{2}\right) \\
& =\frac{\partial}{\partial x_{\alpha}} \rho u_{\alpha} \phi-\frac{\Delta t}{4}\left[\frac{\partial}{\partial x_{\alpha}}\left(\phi \frac{\partial \Pi_{\alpha \beta}^{f \mathrm{col}}}{\partial x_{\beta}}\right)+\frac{\partial}{\partial x_{\beta}}\left(\phi \frac{\partial \Pi_{\alpha \beta}^{f^{\mathrm{col}}}}{\partial x_{\alpha}}\right)\right]+\mathcal{O}\left(\Delta t^{2}\right)
\end{aligned}
$$

Thus, although this scheme is first order corresponding to the flow part $\left(\frac{\partial \Pi_{\alpha \beta}^{\text {fol }}}{\partial x_{\alpha} \partial x_{\beta}}\right)$, it does not introduce any artificial diffusion corresponds to the scalar field. As there is no second order derivative of $\phi$ in this expression. 


\section{Appendix C: Conservative extensions of previously existing scalar transport schemes}

The original scalar transport proposed by Onishi et al.$^{53}$ in Eq.n $(16)$ can be modified for conservative formulation as

$$
\nabla^{O} \cdot(\rho \boldsymbol{u} \phi) \equiv \frac{1}{\Delta t} \sum_{i}\left[\left(f_{i}^{\mathrm{col}} \phi\right)(\boldsymbol{x})-\left(f_{i}^{\mathrm{col}} \phi\right)\left(\boldsymbol{x}+\boldsymbol{c}_{\boldsymbol{i}} \Delta t\right)\right]
$$

It is exactly the same as the proposed upwind operator $\nabla^{U}$, because when considering $\boldsymbol{c}_{\boldsymbol{i}}=-\boldsymbol{c}_{\overline{\boldsymbol{i}}}$, one has

$$
\begin{aligned}
\nabla^{O} \cdot(\rho \boldsymbol{u} \phi) & \equiv \frac{1}{\Delta t} \sum_{i}\left[\left(f_{i}^{\mathrm{col}} \phi\right)(\boldsymbol{x})-\left(f_{\bar{i}}^{\mathrm{col}} \phi\right)\left(\boldsymbol{x}+\boldsymbol{c}_{\boldsymbol{i}} \Delta t\right)\right] \\
& =\frac{1}{\Delta t} \sum_{i}\left[\left(f_{i}^{\mathrm{col}} \phi\right)(\boldsymbol{x})-\left(f_{\bar{i}}^{\mathrm{col}} \phi\right)\left(\boldsymbol{x}-\boldsymbol{c}_{\overline{\boldsymbol{i}}} \Delta t\right)\right] \\
& =\frac{1}{\Delta t} \sum_{i}\left[\left(f_{i}^{\mathrm{col}} \phi\right)(\boldsymbol{x})-\left(f_{i}^{\mathrm{col}} \phi\right)\left(\boldsymbol{x}-\boldsymbol{c}_{\boldsymbol{i}} \Delta t\right)\right] \\
& =\nabla^{U} \cdot(\rho \boldsymbol{u} \phi)
\end{aligned}
$$

The corrected low-dissipating counterpart of this scheme introduced in $\frac{5355}{50}$ in Eq. (17) can be expressed as

$$
\nabla^{O C} \cdot(\rho \boldsymbol{u} \phi) \equiv \frac{1}{\Delta t} \sum_{i}\left\{\left[\left(f_{i}^{\mathrm{col}}-\omega_{i} \sum_{j} f_{j}^{\mathrm{col}}\right) \phi\right](\boldsymbol{x})-\left[\left(f_{\bar{i}}^{\mathrm{col}}-\omega_{i} \sum_{j} f_{j}^{\mathrm{col}}\right) \phi\right]\left(\boldsymbol{x}+\boldsymbol{c}_{\boldsymbol{i}} \Delta t\right)\right\},
$$

with $\omega_{i}$ the Gaussian weights of the lattice. It is worth noting that the $\rho$ is substituted by $\sum_{j} f_{j}^{\text {col }}$ to be more general, thus can be cooperated with the pressure based HRR- $p$ solver.

Finally, in the context of conservative formulations, the scheme proposed by Osmanlic et al. 54 given in Eq. equation $(19)$ can be written with the definition of the mass exchange $\Delta m_{i}$ in Eq. (18)

$$
\nabla^{O S} \cdot(\rho \boldsymbol{u} \phi) \equiv-\sum_{i} \Delta m_{i} \begin{cases}\phi(\boldsymbol{x}, t) & \text { if } \Delta m_{i} \leq 0 \\ \phi\left(\boldsymbol{x}+\boldsymbol{c}_{\boldsymbol{i}} \Delta t, t\right) & \text { otherwise }\end{cases}
$$




\section{Appendix D: Hybrid regularized recursive collision in HRR- $p$}

The D3Q19 rotational symmetry basis of Gauss-Hermite polynomials used in HRR-p reads

$$
\begin{aligned}
& \mathcal{H}_{i}^{(0)} \equiv 1 \\
& \mathcal{H}_{i, \alpha}^{(1)} \equiv c_{i \alpha} \\
& \mathcal{H}_{i, \alpha \beta}^{(2)} \equiv c_{i \alpha} c_{i \beta}-c_{s}^{2} \delta_{\alpha \beta} \\
& \mathcal{H}_{i, \alpha \beta \gamma}^{(3)} \equiv c_{i \alpha} c_{i \beta} c_{i \gamma}-c_{s}^{2}\left(\delta_{\alpha \beta} c_{i \gamma}+\delta_{\beta \gamma} c_{i \alpha}+\delta_{\alpha \gamma} c_{i \beta}\right) \\
& \mathcal{H}_{i, 1}^{(3 r)} \equiv \mathcal{H}_{i, x x y}^{(3)}+\mathcal{H}_{i, y z z}^{(3)} \\
& \mathcal{H}_{i, 2}^{(3 r)} \equiv \mathcal{H}_{i, x z z}^{(3)}+\mathcal{H}_{i, x y y}^{(3)} \\
& \mathcal{H}_{i, 3}^{(3 r)} \equiv \mathcal{H}_{i, y y z}^{(3)}+\mathcal{H}_{i, x x z}^{(3)} \\
& \mathcal{H}_{i, 4}^{(3 r)} \equiv \mathcal{H}_{i, x x y}^{(3)}-\mathcal{H}_{i, y z z}^{(3)} \\
& \mathcal{H}_{i, 5}^{(3 r)} \equiv \mathcal{H}_{i, x z z}^{(3)}-\mathcal{H}_{i, x y y}^{(3)} \\
& \mathcal{H}_{i, 6}^{(3 r)} \equiv \mathcal{H}_{i, y y z}^{(3)}-\mathcal{H}_{i, x x z}^{(3)}
\end{aligned}
$$

In the collision operation (eq. (27)), the projection of the equilibrium distribution on the Hermite basis reads

$$
\begin{aligned}
& a^{(0), \mathrm{eq}} \equiv \mathcal{H}_{i}^{(0)} f_{i}^{\mathrm{eq}}=\frac{p}{c_{s}^{2}} \\
& a_{\alpha}^{(1), \mathrm{eq}} \equiv \mathcal{H}_{i, \alpha}^{(1)} f_{i}^{\mathrm{eq}}=\rho u_{\alpha} \\
& a_{\alpha \beta}^{(2), \mathrm{eq}} \equiv \mathcal{H}_{i, \alpha \beta}^{(2)} f_{i}^{\mathrm{eq}}=\rho u_{\alpha} u_{\beta} \\
& a_{\alpha \beta \gamma}^{(3), \mathrm{eq}} \equiv \mathcal{H}_{i, \alpha \beta \gamma}^{(3)} f_{i}^{\mathrm{eq}}=\rho u_{\alpha} u_{\beta} u_{\gamma} \\
& a_{1}^{(3 r), \mathrm{eq}} \equiv 3\left(a_{x x y}^{(3), \mathrm{eq}}+a_{y z z}^{(3), \mathrm{eq}}\right) \\
& a_{2}^{(3 r), \mathrm{eq}} \equiv 3\left(a_{x z z}^{(3), \mathrm{eq}}+a_{x y y}^{(3), \mathrm{eq}}\right) \\
& a_{3}^{(3 r), \mathrm{eq}} \equiv 3\left(a_{y y z}^{(3), \mathrm{eq}}+a_{x x z}^{(3), \mathrm{eq}}\right) \\
& a_{4}^{(3 r), \mathrm{eq}} \equiv a_{x x y}^{(3), \mathrm{eq}}-a_{y z z}^{(3), \mathrm{eq}} \\
& a_{5}^{(3 r), \mathrm{eq}} \equiv a_{x z z}^{(3), \mathrm{eq}}-a_{x y y}^{(3), \mathrm{eq}} \\
& a_{6}^{(3 r), \mathrm{eq}} \equiv a_{y y z}^{(3), \mathrm{eq}}-a_{x x z}^{(3), \mathrm{eq}}
\end{aligned}
$$

The second order projections of the non-equilibrium part are evaluated as

$$
a_{\alpha \beta}^{(2), \text { neq }}=\mathcal{H}_{i \alpha \beta}^{(2)}\left[f_{i}^{\mathrm{col}}\left(\boldsymbol{x}-\boldsymbol{c}_{\boldsymbol{i}} \Delta t, t-\Delta t\right)-f_{i}^{\mathrm{eq}}\right] .
$$


The corresponding third order projections are calculated through a recursive procedure

$$
a_{\alpha \beta \gamma}^{(3) \text {,neq }}=u_{\alpha} a_{\beta \gamma}^{\text {neq }}+u_{\beta} a_{\alpha \gamma}^{\text {neq }}+u_{\gamma} a_{\alpha \beta}^{\text {neq }} .
$$

The forcing term $F_{i}^{E}$ in the collision step is added to obtain a correct viscous tensor. In the current study, it is evaluated as described in $\underline{58}$.

\section{Appendix E: Numerical conservation of mass and momentum in HRR- $p$}

In any periodic calculation domains, the spacial integration of density at next time step evaluated by HRR- $p$ can be written as

$$
\begin{aligned}
\sum_{\boldsymbol{x}} \rho(\boldsymbol{x}, t+\Delta t) & =\sum_{\boldsymbol{x}}\left[\rho+\sum_{i}\left(f_{i}^{\mathrm{col}^{-}}-f_{i}^{\mathrm{col}}\right)\right] \\
& =\sum_{\boldsymbol{x}} \rho+\sum_{i} \sum_{\boldsymbol{x}}\left(f_{i}^{\mathrm{col}^{-}}-f_{i}^{\mathrm{col}}\right) \\
& =\sum_{\boldsymbol{x}} \rho
\end{aligned}
$$

which is the same as the current time step. The momentum components at next time step

$$
\begin{aligned}
\sum_{\boldsymbol{x}} \rho u_{\alpha}(\boldsymbol{x}, t+\Delta t) & =\sum_{\boldsymbol{x}} \sum_{i}\left(c_{i \alpha} f_{i}^{\mathrm{col}^{-}}\right) \\
& =\sum_{\boldsymbol{x}} \sum_{i}\left(c_{i \alpha} f_{i}^{\mathrm{col}}\right) \\
& =\sum_{\boldsymbol{x}} \rho u_{\alpha}
\end{aligned}
$$

which is also identical to the corresponding integration in the current time step.

\section{REFERENCES}

${ }^{1}$ J. Stolnick, A. Khodadoust, J. Alonso, D. Darmfold, W. Gropp, E. Lurie, and D. Mavriplis. Cfd vision 2030 study: A path to revolutionary computational aerosciences. NASA/CR2014-218178, 2014.

${ }^{2} \mathrm{Z}$. Guo and C. Shu. The Lattice Boltzmann Method and its applications in engineering. World Scientific, 2013.

${ }^{3}$ T. Krüger, H. Kusumaatmaja, A. Kuzmin, O. Shardt, G. Silva, and E.M. Viggen. The Lattice Boltzmann Method. Principles and Practice. Springer, 2017. 
${ }^{4}$ F. Filbet and S. Jin. A class of asymptotic-preserving schemes for kinetic equations and related problems with stiff sources. J. Comput. Phys., 229:7625-7648, 2010.

${ }^{5} \mathrm{~S}$. Jin. Asymptotic preserving (ap) schemes for multiscale kinetic and hyperbolic equations. Riv. Mat. Univ. Parma, 3:177-216, 2012.

${ }^{6}$ M. Bennoune, M. Lemou, and L. Mieussens. Uniformly stable numerical schemes for the boltzmann equation preserving the compressible navier-stokes asymptotics. J. Comput. Phys., 227:3781-3803, 2008.

${ }^{7} \mathrm{~S}$. Chen and K. Xu. A comparative study of an asymptotic preserving scheme and unified gas-kinetic scheme in continuum flow limit. J. Comput. Phys., 288:52-65, 2015.

${ }^{8}$ V.I. Kolobov, R.R. Arslanbekov, V.V. Aristov, A.A. Frolova, and S.A. Zabelok. Unified solver for rarefied and continuum flows with adaptive mesh and algorithm refinement. $J$. Comput. Phys., 223:589-608, 2007.

${ }^{9}$ L.M. Yang, C. Shu, Y. Wang, and Y. Sun. Development of discrete gas kinetic scheme for simulation of $3 \mathrm{~d}$ viscous incompressible and compressible flows. J. Comput. Phys., 319:129-144, 2016.

${ }^{10}$ X. Shan, X.F. Yuan, and H. Chen. Kinetic theory representation of hydrodynamics: a way beyond the navier-stokes equation. J. Fluid Mech., 550:413-441, 2006.

${ }^{11}$ M. Watari. Finite difference lattice boltzmann method with arbitrary specific heat ratio applicable to supersonic flow simulations. Physica A, 382:502-522, 2007.

${ }^{12}$ J. Zhang, G. Yan, X. Shi, and Y. Dong. A lattice boltzmann model for the compressible euler equations with second-order acuracy. Int. J. Numer. Methods Fluids, 60:95-117, 2009.

${ }^{13}$ Y.B. Gan, A.G. Xu, G.C. Zhang, and Y.J. Li. Flux limiter lattice boltzmann scheme approach to compressible flows with flexible specific-heat ratio and prandtl number. Commun. Theor. Phys., 56:1-14, 2017.

${ }^{14} \mathrm{~T}$. Kataoka and T. Hanada. New lattice boltzmann model for the compressible navierstokes equations. Int. J. Numer. Methods Fluids, 91:183-197, 2019.

${ }^{15}$ Y. Gan, A. Xu, G. Zhang, and S. Succi. Discrete boltzmann trans-scale modeling of high-speed compressible flows. Phys. Rev. E, 97:053312, 2018.

${ }^{16}$ M. Atif, M. Namburi, and S. Ansumali. Higher-order lattice boltzmann model for thermodynamics. Phys. Rev. E, 98:053311, 2018. 
${ }^{17}$ E. Fares, M. Wessels, Y. Li, P. Gopalakrishnan, R. Zhang, C. Sun, N. Gopalaswamy, P. Roberts, J. Hoch, and H. Chen. Validation of a lattice-boltzmann approach for transonic and supersonic flow simulations. AIAA Paper, 2014-0952, 2014.

${ }^{18}$ Y. Feng, P. Sagaut, and W.Q. Tao. A compressible lattice boltzmann finite volume model for high subsonic and transonic flows on regular lattices. Computers and Fluids, 131:45-55, 2016.

${ }^{19}$ Y. Feng, P. Sagaut, and W.Q. Tao. A three dimensional lattice model for thermal compressible flow on standard lattices. J. Comput. Phys., 303:514-529, 2015.

${ }^{20}$ N. Frapolli, S.S. Chikatamarla, and I.V. Karlin. Entropic lattice boltzmann model for compressible flows. Phys. Rev. E, 92:061301(R), 2015.

${ }^{21}$ M.H. Saadat, F. Bösch, and I.V. Karlin. Lattice boltzman model for compressible flows on standard latices: variable prandtl number and adiabatic exponent. Phys. Rev. E, 99:0, 2017.

${ }^{22}$ Y. Feng, S. Guo, W.Q. Tao, and P. Sagaut. Regularized thermal lattice boltzmann method for natural convection with large temperature differences. Int. J. Heat Mass Transfer, 125:1379-1391, 2018.

${ }^{23}$ Yongliang Feng, Pierre Boivin, Jérôme Jacob, and Pierre Sagaut. Hybrid recursive regularized thermal lattice boltzmann model for high subsonic compressible flows. Journal of Computational Physics, 394:82 - 99, 2019.

${ }^{24}$ Y. Feng, P. Boivin, J. Jacob, and P. Sagaut. Hybrid recursive regularized lattice boltzmann simulation of humid air with application to meteorological flows. Physical Review E, 100(2):023304, 2019.

${ }^{25}$ V.V. Aristov and F.G. Cheremisin. The conservative splitting method for solving boltzmann's equation. USSR Comput. Maths. Math. Phys., 20:208-225, 1980.

${ }^{26}$ V.A. Titarev. Conservative numerical methods for model kinetic equations. Computers and Fluids, 36:1446-1459, 2007.

${ }^{27}$ L. Mieussens. Discrete-velocity models and numerical schemes for the boltzmann-bgk equation in plane and axisymmetric geometries. J. Comput. Phys., 162:429-466, 2000.

${ }^{28}$ S. Pieraccini and G. Puppo. Implicit-explicit schmes for bgk kinetic equations. J. Sci. Comput., 32:1-28, 2007.

${ }^{29}$ J. Latt, C. Coreixas, J. Beny, and A. Parmigiani. Efficient supersonic flows through higherorder guided equilibrium with lattice boltzmann. Phil. Trans. R. Soc. A, 378:20190559, 
2020.

${ }^{30}$ R.F. Qiu, C.X. Zhu, R.Q. Chen, J.F. Zhu, and Y.C. You. A double-distribution-function lattice boltzmann model for high-speed compressible viscous flows. Computers and Fluids, $166: 24-31,2018$.

${ }^{31}$ S. Guo, Y. Feng, and P. Sagaut. Evaluation on formulation of energy conservation equation in hybrid compressible lattice boltzmann method. submitted, 2020.

${ }^{32}$ R.H. Sanders and K.H. Prendergast. The possible relation of the 3 -kiloparsec arm to explosions in the galactic nucleus. Astrophys. J., 188:489-500, 1974.

${ }^{33}$ D.I. Pullin. Direct simulation methods for compressible inviscid ideal-gas flow. J. Comput. Phys., 34:231-244, 1980.

${ }^{34}$ Benoit Perthame. An Introduction to Kinetic Schemes for Gas Dynamics, pages 1-27. Springer Berlin Heidelberg, Berlin, Heidelberg, 1999.

${ }^{35}$ K. Xu and K.H. Prendergast. Numerical navier-stokes solutions from gas kinetic theory. J. Comput. Phys., 114:9-17, 1994.

${ }^{36} \mathrm{Kun} \mathrm{Xu}$ and Antony Jameson. Gas-kinetic relaxation (bgk-type) schemes for the compressible euler equations. page 1736, 1995.

${ }^{37}$ Kun XU. Direct modelling for Computational Fluid Dynamics. World Scientific, 2015.

${ }^{38} \mathrm{~K} . \mathrm{Xu}$ and J.H. Huang. A unified gas-kinetic scheme for continuum and rarefied flows. J. Comput. Phys., 229:7747-7764, 2010.

${ }^{39} \mathrm{~K}$. Xu and J.H. Huang. An improved unified gas-kinetic scheme and the study of shock structures. IMA J. Applied Mathematics, 76:698-711, 2011.

${ }^{40}$ S. Chen, K. Xu, C. Lee, and Q. Cai. A unified gas-kinetic scheme with moving mesh and velocity space adaptation. J. Comput. Phys., 231:6643-6664, 2012.

${ }^{41}$ Z. Guo, K. Xu, and R. Wang. Discrete unified gas kinetic scheme for all knudsen flows: Low-speed isothermal case. Phys. Rev. E, 88:033305, 2013.

${ }^{42}$ Z. Guo, R. Wang, and K. Xu. Discrete unified gas kinetic scheme for all knudsen flows. ii. thermal compressible case. Phys. Rev. E, 91:033313, 2015.

${ }^{43}$ L. Zhu, Z. Guo, and K. Xu. Discrete unified gas kinetic scheme on unstructured meshes. Computers and Fluids, 127:211-225, 2016.

${ }^{44}$ L.Q. Zhang, Z. Chen, L.M. Yang, and C. Shu. Double distribution function-based discrete gas kinetic scheme for viscous incompressible and compressible flows. J. Comput. Phys., 412:109428, 2020. 
${ }^{45}$ L.J. Xuan and K. Xu. An efficient high-order finite difference gas-kinetic scheme for the euler and navier-stokes equations. Computers and Fluids, 166:243-252, 2018.

${ }^{46}$ L.M. Yang, C. Shu, and J. Wu. A moment conservation-based non-free parameter compressible lattice boltzmann model and its application for flux evaluation at cell interface. Computers and Fluids, 79:190-199, 2013.

${ }^{47}$ Y. Wang, C. Shu, and C.J. Teo. Development of lbgk and incompressible lbgk-based lattice boltzmann flux solvers for simulation of incompressible flows. Int. J. Numer. Methods Fluids, 75:344-364, 2014.

${ }^{48}$ Y. Wang, L. Yang, and C. Shu. From lattice boltzmann method to lattice boltzmann flux solver. Entropy, 17:7713-7735, 2015.

${ }^{49}$ Z. Chen, C. Shu, D. Tan, and C. Wu. On improvements of simplified and highly stable lattice boltzmann method: Formulations, boundary treatment, and stability analysis. Int. J. Numer. Methods Fluids, pages 1-19, 2018.

${ }^{50}$ Z. Chen, C. Shu, and D. Tan. Three-dimensional simplified and unconditionally stable lattice boltzman method for incompressible isothermal and thermal flows. Phys. Fluids, 29:053601, 2017.

${ }^{51}$ Z. Chen, C. Shu, and D. Tan. A simplified thermal lattice boltzman method without evolution of distribution functions. Int. J. Heat Mass Transfer, 105:741-757, 2017.

${ }^{52}$ Z. Chen, C. Shu, Y. Wang, L.M. Yang, and D. Tan. A simplified lattice boltzman method without evolution of distribution function. Adv. Appl. Math. Mech., 9:1-22, 2017.

${ }^{53}$ Junya Onishi, Yu Chen, and Hirotada Ohashi. A lattice boltzmann model for polymeric liquids. Progress in Computational Fluid Dynamics, an International Journal, 5(1-2):7584, 2005.

${ }^{54}$ F Osmanlic and C Körner. Lattice boltzmann method for oldroyd-b fluids. Computers $\&$ Fluids, 124:190-196, 2016.

${ }^{55}$ VE Küng, F Osmanlic, M. Markl, and C. Körner. Comparison of passive scalar transport models coupled with the lattice boltzmann method. Computers $\&$ Mathematics with Applications, 79(1):55-65, 2020.

${ }^{56}$ G.V. Krivovichev. Analysis of the parametric models of passive scalar transport used in the lattice boltzmann method. Computers and Mathematics with Applications, 79:1503-1524, 2020 . 
${ }^{57}$ G.V. Krivovichev. Parametric schemes for the simulation of advection process in finitedifference-based single-relaxation-time lattice boltzmann methods. Journal of Computational Science, 44:101151, 2020.

${ }^{58}$ G. Farag, S. Zhao, T. Coratger, P. Boivin, G. Chiavassa, and P. Sagaut. A pressure-based regularized lattice-boltzmann method for the simulation of compressible flows. Physics of Fluids, 32(6):066106, 2020.

${ }^{59}$ Jérôme Jacob, Orestis Malaspinas, and Pierre Sagaut. A new hybrid recursive regularised bhatnagar-gross-krook collision model for lattice boltzmann method-based large eddy simulation. Journal of Turbulence, 19(11-12):1051-1076, 2018.

${ }^{60}$ M. Tayyab, S. Zhao, Y. Feng, and P. Boivin. Hybrid regularized lattice-boltzmann modelling of premixed and non-premixed combustion processes. Combustion and Flame, 211:173 - 184, 2020.

${ }^{61}$ G. Coppola, F. Capuano, S. Pirozzoli, and L. de Luca. Numerically stable formulations of convective terms for turbulent compressible flows. Journal of Computational Physics, $382: 86-104,2019$.

${ }^{62}$ Chi-Wang Shu, Wai-Sun Don, David Gottlieb, Oleg Schilling, and Leland Jameson. Numerical convergence study of nearly incompressible, inviscid taylor-green vortex flow. Journal of Scientific Computing, 24(1):1-27, 2005.

${ }^{63}$ Naifu Peng and Yue Yang. Effects of the mach number on the evolution of vortex-surface fields in compressible taylor-green flows. Phys. Rev. Fluids, 3:013401, Jan 2018.

${ }^{64}$ Yongliang Feng, Muhammad Tayyab, and Pierre Boivin. A lattice-boltzmann model for low-mach reactive flows. Combustion and Flame, 196:249 - 254, 2018.

${ }^{65}$ Yongliang Feng, S Guo, Jérôme Jacob, and Pierre Sagaut. Solid wall and open boundary conditions in hybrid recursive regularized lattice boltzmann method for compressible flows. Physics of Fluids, 31(12):126103, 2019.

${ }^{66}$ Antony Jameson, Wolfgang Schmidt, and Eli Turkel. Numerical solution of the euler equations by finite volume methods using runge kutta time stepping schemes. In 14 th fluid and plasma dynamics conference, page 1259, 1981.

${ }^{67}$ Eleuterio F Toro. Riemann solvers and numerical methods for fluid dynamics: a practical introduction. Springer Science \& Business Media, 2009.

${ }^{68}$ Peter D. Lax and Liu Xu-Dong. Solution of two-dimensional riemann problems of gas dynamics by positive schemes. SIAM Journal on Scientific Computing, 19(2):319-340, 
1998. 